
T1/2 194

PPPL-2947

UC- $420,426,427$

\section{THE GENERALIZED ACCESSIBILITY AND SPECTRAL GAP OF LOWER HYBRID WAVES IN TOKAMAKS}

MARCH, 1994

\section{BY} \\ H. TAKAHASHI
}




\section{NOTICE}

This report was prepared as an account of work sponsored by an agency of the United States Government. Neither the United States Government nor any agency thereof, nor any of their employees, makes any warranty, express or implied, or assumes any legal liability or responsibility for the accuracy, completeness, or usefulness of any information, apparatus, product, or process disclosed, or represents that its use would not infringe privately owned rights. Reference herein to any specific commercial produce, process, or service by trade name, trademark, manufacturer, or otherwise, does not necessarily constitute or imply its endorsement, recommendation, or favoring by the United States Government or any agency thereof. The views and opinions of authors expressed herein do not necessarily state or reflect those of the United States Government or any agency thereof.

\section{NOTICE}

This report has been reproduced from the best available copy. Available in paper copy and microfiche.

Number of pages in this report: 45

DOE and DOE contractors can obtain copies of this report from:

Office of Scientific and Technical Information

P.O. Box 62

Oak Ridge, TN 37831;

(615) $576-8401$.

This report is publicly available from the:

National Technical Information Service

Department of Commerce

5285 Port Royal Road

Springfield, Virginia 22161

(703) $487-4650$ 


\title{
THE GENERALIZED ACCESSIBILITY AND SPECTRAL GAP OF LOWER HYBRID WAVES IN TOKAMAKS
}

\author{
Hironori Takahashi \\ Princeton Plasma Physics Laboratory, Princeton University \\ P.O. Box 451, 'Princeton, New Jersey 08544
}

\begin{abstract}
The generalized accessibility of lower hybrid waves, primarily in the current drive regime of tokamak plasmas, which may include shifting, either upward or downward, of the parallel refractive index $\left(n_{\|}\right)$, is investigated, based upon a cold plasma dispersion relation and various geometrical constraint (G.C.) relations imposed on the behavior of $n_{\|}$. It is shown that $n_{\|}$upshifting can be bounded and insufficient to bridge a large spectral gap to cause wave damping, depending upon whether the G.C. relation allows the oblique resonance to occur. The traditional $n_{\|}$upshifting mechanism caused by the pitch angle of magretic field lines is shown to lead to contradictions with experimental observations. An upshifting mechanism brought about by the density gradient along field lines is proposed, which is not inconsistent with experimental observations, and provides plausible explanations to some unresolved issues of lower hybrid wave theory, including generation of 'seed electrons.'
\end{abstract}

\section{INTRODUCTION}

The efficacy of Lower Hybrid Current Drive ${ }^{1}$ (LHCD) in driving a bulk of the plasma current in the tokamak has been well established (see review articles ${ }^{2-4}$ ). The emphasis in the application of LHCD has recently turned to subtle modifications of the radial profile of the plasma current for enhanced plasma performance. ${ }^{5-9}$ Controlled current profile modifications are thought to be achievable by allowing waves to propagate to a desired location, damp, and generate a current there. Thorough understanding of wave propagation and damping mechanisms is essential in achieving these goals.

The term, 'accessibility,' originally referred to the question of whether or not the lower hybrid resonance layer was reachable, i.e., whether or not waves having a wavelength imposed by an antenna can propagate unhindered to a location where the lower hybrid resonance condition is met and ion heating is thought to occur. As the scope of research involving lower hybrid waves broadened to include conditions under which no lower hybrid resonance exists within the plasma, such as electron heating regime and current drive regime, ${ }^{3}$ the same term came to mean whether the plasma center is reachable. In the context of current profile modifications, the accessibility now means whether or not any specified point within the plasma is reachable. There are usually some constraints imposed upon some aspects of the waves in determining the accessibility. The accessibility analysis is classified in this article depending upon constraints placed on the wavelength parallel to the magnetic field lines: in the 'classical' accessibility, the parallel wavelength is assumed fixed, e.g., through the periodicity or boundary conditions,

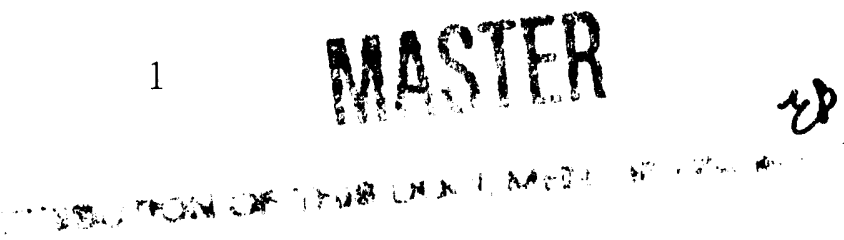


and in the 'generalized' accessibility, changes in the parallel wavelength is permitted. This article is concerned mostly with the generalized accessibility, but its relationship to the classical accessibility is also examined.

In a simple-minded picture of the classical accessibility involving the lower hybrid resonance, the resonance is a special condition that occurs at a specific location in an inhornogeneous plasma, and waves launched from the plasma edge propagate through intervening regions to reach the resonance layer where the waves are absorbed. An examination of the cold plasma dispersion relation shows, however, a resonance exists, in the form of an 'oblique' resonance, everywhere in the intervening regions between (almost) the plasma edge and the lower hybrid resonance layer. The lower hybrid resonance is simply a limiting form of the oblique resonance that occurs at a right angle to the magnetic field lines. The oblique resonance is, however, precluded under the constraints of the widely employed classical accessibility analysis, because the obliqueness requires both the parallel and perpendicular wavelengths to become very short while maintaining a fixed ratio between them. Perhaps for this reason, the notion of the oblique resonance has remained obscure. The oblique resonance can occur in the generalized accessibility depending upon conditions.

It is traditionally assumed in the analysis of lower hybrid wave propagation in an inhomogeneous tokamak plasma that no variations in zero-order parameters occur in the toroidal direction, and the toroidal mode number of waves (i.e., the number of whole wavelengths) is an 'invariant of the motion.' Magnetic field lines in the tokamak are generally not in the purely toroidal direction because of the presence of poloidal fields. The component, $n_{\|}$, of the refractive index vector parallel to the magnetic field direction therefore does not have to remain fixed. The invariance of toroidal mode number nevertheless imposes a constraint on the manner $n_{\|}$varies, because the tokamak magnetic geometry ties the toroidal and parallel components of the refractive index vector. A significant feature of this 'geometrical constraint,' recognized by Schuss, et al. ${ }^{10}$ (in a cylindrical geometry analysis), is that $n_{\|}$can increase ('upshift') or decrease ('downshift'), if the perpendicular refractive index, $n_{\perp}$, which is usually much larger than $r_{\|}$, beconies rotated about the field line, and projects a component in the parallel direction. Determínation of lower hybrid wave propagation and damping in an inhomogeneous plasma must usually rely on numerical techniques such as ray tracing analysis. ${ }^{11}$ Upshifting and downshifting of $n_{\|}$have been demonstrated in theoretical analyses ${ }^{2,12-16}$ using the ray tracing technique in the tokamak geometry. It will be shown in the present article that the geometrical constraint arising from the toroidal mode number invariance can also allow an oblique resonance to occur. This global constraint on the waves will, however, prove to lead to predictions that are in contradiction with experimental observations. A new local geometrical constraint will be introduced, and its ramifications on some unresolved issues of lower hybrid wave theory will be discussed.

There is an aspect of wave damping mechanism that has not been fully understood. The lower hybrid waves in the current drive regime are theoreticall expected to damp through Landau damping by resonantly interacting with electrons that are moving at speeds near the wave phase speed parallel to the confining magnetic field. The spectrum of waves launched into a tokamak plasma by an LHCD wave antenna ('grill') has, however, a phase speed often 
much greater than the thermal speed of electrons, and there are few electrons that are resonant with the waves. This gap between the parallel phase speed of launched waves and electron thermal speed is commonly known as the 'spectral gap.' Upshifting of $n_{\|}$may be able to fill this gap, and cause the waves to damp. Although a direct experimental confirmation of $n_{\|}$ upshifting is difficult, it has nevertheless become widely accepted as an explanation for how the lower hybrid waves damp in spite of the spectral gap. The spectral gap can be large or small depending upon the wave phase speed and electron temperature. Implicit in invoking $n_{\|}$upshifting to explain the wave damping under any conditions is an assumption that the $n_{\|}$ upshifting can be arbitrarily large to fill a spectral gap of any size. If there is a limit to the upshifting, the explanation can fail under some circumstances.

Some steps of the development of lower hybrid wave propagation theory will be revisited, first, to introduce some definitions and features of plasmas under consideration, and, second, to clarify the connection between the classical and generalized accessibilities. Waves in a cold plasma in LHCD regime are then examined in terms of wave normal surfaces. The oblique resonance is a distinguishing feature of a lemniscoid wave normal surface that characterizes lower hybrid waves. Relationships between the oblique resonance and some well-known concepts in the lower hybrid theory, such as the electrostatic approximation, are examined. An analytical expression is derived for the global geometrical constraint based upon the toroidal mode number invariance. The electrostatic approximation and geometrical constraint are solved together to arrive at a range of solutions admissible under the combined requirements of the dispersion relation and toroidal mode number invariance. The 'admissible solutions' will show that characters of wave propagation are qualitatively different depending upon the realizability of the oblique resonance. When the oblique resonance is realizable, $n_{\|}$upshifting is unlinited, and can in principle bridge spectral gaps of any size. Furthermore, toroidal reflection of waves is possible. When the oblique resonance is unrealizable, there is an upper bound to $n_{\|}$upshifting, and spectral gaps may not be filled depending upon conditions. An analytical expression for the bound to the $n_{\|}$"pshifting, when it exists, is derived. Notions of 'wave domain' and 'damping domain' are introduced. The gap between the two domains, which may exist when upshift ing is bounded, is a spectral gap that cannot be bridged through $n_{\|}$upshifting.

The analytical expression, reported earlier in a simpler form ${ }^{17}$ and derived in detail in the present article, for admissible solutions shows that $n_{\|}$upshifting can be bounded or unbounded, depending primarily upon the plasma density (in a dimensionless sense) and the pitch angle of magnetic field lines. As an example of a tokamak that has a small aspect ratio and consequently a large field-line pitch angle, TORE SUPRA is considered. It operates at comparatively high densities. It has unbounded $n_{\|}$upshifting over most of the plasma volume. Shaping of the plasma cross section also results, to a lesser extent, in a large field-line pitch angle. The PBX$\mathrm{M}$ tokamak, with a medium aspect ratio, operates with or without cross-sectional shaping, and at moderate densities. It has bounded $n_{\|}$upshifting, but the upper bound can range from being nearly sufficient to insufficient for wave damping. The TRIAM-1M tokamak represents an opposite extreme: it has a large aspect ratio, small plasma current, and high toroidal field, and consequently a small field-line pitch angle. It also operates at low densities. Essentially no $n_{\|}$upshifting occurs and no wave damping is theoretically expected. In all of these tokamaks, however, there was experimental evidence that a current was driven by LHCD. The TRIAM- 
$1 \mathrm{M}$ discharges were in fact entirely sustained by LHCD. The $n_{\|}$upshifting caused by the pitch angle of field lines will thus lead to contradictions with experimental evidence.

The oblique resonance can be realizable, and $n_{\|}$upshifting can be unbounded, when there is a sufficiently large density gradient along magnetic field lines. The total density gradient must be 'tilted' into a direction parallel to field lines by an angle commensurate with the oblique resonance angle. A key assumption here is that the component of the refractive index vector in a direction perpendicular to the density gradient vector remains invariant. It was shown by Briggs and Parker ${ }^{18}$ in a plane-stratified geometry that there is a 'new absorption layer' when the density gradient is tilted from the perpendicular to the field lines. We will show that their 'new absorption layer' is actually a manifestation of the oblique resonance, and that the density gradient tilting will lead to $n_{\|}$upshifting in the tokamak geometry. The density gradient along the field lines may be present in the equilibrium state under special circumstances, or may be produced locally and temporarily by density fluctuations or MHD perturbations. Tilt angles large enough to make the oblique resonance realizable can be produced easily when the perpendicular gradient is also reduced under these conditions. This $n_{\|}$upshifting mechanism is thus a candidate to explain experimentally observed lower hybrid wave damping in the face of spectral gaps.

Past ion heating and current drive) experiments, and more recent profile modification experiments have left some fundamental) issues of lower hybrid wave physics unresolved. They include among others the lack of consistent observation of ion heating, ${ }^{3}$ and the existence of a density limit in the current drive efficacy. In connection with possible mechanisms to fill the spectral gap, the existence of 'seed electrons' is often postulated, but the origin of these electrons has remained unspecified. We will discuss how the notion of the oblique resonance and the proposed $n_{\|}$upshifting mechanism based upon the parallel density gradient may offer plausible qualitative explanations to these issues.

In Sec. II, model plasmas are described with parameters spanning a wide range of LHCD experiments in tokamaks. In Sec. III, nature of the solutions to a cold plasma dispersion relation are discussed. In Sec. III-A, definitions of the dielectric tensor elements are given. Their spatial variations are examined. In Sec. III-B, the classical accessibility of waves with a fixed $n_{\|}$into an inhomogeneous plasma is discussed. In Sec. III-C, the notions of wave normal surfaces and oblique resonance are introduced. In Sec. III-D, the notions of inverse wave normal surfaces and electrostatic approximation are discussed. In Sec. IV, a constraint placed on $n_{\|}$, arising from the toroidal mode number invariance assumption, is derived. In Sec. V, a concept of the range of solutions, admissible under the combined requirements of the dispersion relation and geometrical constraint relation, is discussed. In Sec. V-A, a geometrical interpretation of the admissible solutions is given. In Sec. V-B, an analytical expression for the range of admissible solutions is derived. In Sec. V-C, a notion of the wave domain is introduced. The spectral gap and wave damping are discussed. In Sec. V-D $n_{\|}$upshifting caused by the parallel density gradient is proposed. In Sec. VI, a summary and discussion are presented. 


\section{PLASMA MODELS}

Model plasmas are employed to obtain numerical examples. We consider for most part a bean-shaped model plasma, similar to plasmas in LHCD experiments on the PBX-M tokamak. This model plasma, referred to as 'PBXM,' is representative of tokamaks in which $n_{\|}$upshifting is bounded according to the 'traditional' mechanism based upon the field-line pitch angle, but the upper bound of $n_{\|}$is large enough for the waves to satisfy (or nearly satisfy) the damping conditions. Detailed calculations based upon equilibria reconstructed for experimental plasmas in this tokamak is reported elsewhere. ${ }^{19}$ A second model, referred to as 'TRIAM,' is a circular plasma similar to those sustained entirely by LHCD (i.e., no inductive drive) in the TRIAM$1 \mathrm{M}$ tokamak, ${ }^{20}$ and is representative of tokamaks in which the $n_{\|}$upshifting is severely limited, and wave damping through traditional $n_{\|}$upshifting is highly unlikely. A third model, referred to as 'SUPRA,' is a circular plasma similar to those found in the TORE SUPRA tokamak, ${ }^{16}$ and is representative of tokamaks in which traditional $n_{\|}$upshifting is unbounded over most of the plasma cross section. Some basic parameters of the models are tabulated in Table I. Numerical examples discussed in later sections are based upon these sets of parameters unless specifically stated otherwise.

Table 1: Parameters of Plasma Models - I

\begin{tabular}{|r|r|r|r|l|}
\hline & TRIAM & PBXM & SUPRA & Units \\
\hline$B_{\phi}$ & 7 & 1.5 & 3.9 & $\mathrm{~T}$ \\
$n_{e}(0)$ & 2 & 3 & 4 & $10^{19} / \mathrm{m}^{3}$ \\
$T_{e}(0)$ & 0.6 & 1.5 & 1 & $\mathrm{keV}$ \\
$f_{w}$ & 8.2 & 4.6 & 3.7 & $\mathrm{GHz}$ \\
\hline$I_{p}$ & 40 & 180 & 1,600 & $\mathrm{kA}$ \\
$R_{0}$ & 0.84 & 1.65 & 2.40 & $\mathrm{~m}$ \\
$a_{p}$ & 0.12 & 0.3 & 0.8 & $\mathrm{~m}$ \\
$\mathcal{A}$ & 7.0 & 5.5 & 3.0 & $\mathrm{~m}$ \\
$q_{e d g e}$ & 15 & 8.3 & 3.3 & \\
\hline$R_{m a j}^{g r}$ & 0.96 & 1.95 & 3.20 & $\mathrm{~m}$ \\
$N_{\phi}^{g r}$ & 297 & 395 & 446 & \\
$n_{\phi}^{g r}$ & 1.8 & 2.1 & 1.8 & \\
\hline
\end{tabular}

The major and minor radial coordinates are $R_{m a j}$ and $r_{m i n}$, respectively. The plasma major radius is designated by $R_{0}$. For the circular plasmas, the minor radius is designated by $a_{p}$. The aspect ratio is defined by $\mathcal{A} \equiv R_{0} / a_{p}$. The electron density is assumed to have a parabolic profile in the normalized minor radius, $n_{e}\left(r_{\min }\right)=n_{e}(0)\left(1-\left(r_{\min } / a_{p}\right)^{2}\right)$. The electron temperature is assumed to have a profile of a parabola raised to the power of $2, T_{e}=$ $T_{e}(0)\left(1-\left(r_{\min } / a_{p}\right)^{2}\right)^{2}$. For the bean-shaped plasma, the 'minor radius' refers to the plasma half-width along the midplane, and the profiles are specified along the midplane. The plasma ion species is chosen to be deuterium for all model plasmas, but the choice is inconsequential in the present analysis.

We classify properties of a model into three categories, and refer to each of them by a 
specific term. We refer to the electron density and temperature, and the intensity (but not the direction) of the total magnetic.field $\left(B_{T}\right)$ as 'plasma properties.' We also regard the wave frequency $\left(f_{w}\right)$ as a plasma property, because we make the density and magnetic field intensity dimensionless using the wave angular frequency $\left(\omega \equiv 2 \pi f_{w}\right)$. The plasma properties determine the local dispersion relation and wave damping. The pitch angle between the magnetic field vector and the toroidal direction plays an important role in determining the geometrical constraint on $n_{\|}$shifting. We refer to parameters that influence the angle as 'magnetic geometry' properties. They include the plasma current $\left(I_{p}\right)$ and toroidal magnetic field $\left(B_{\phi}\right)$ as well as geometrical properties of the plasma, such as the plasma shape, and aspect ratio. Finally, we refer to the major radial location $\left(R_{m a j}^{g r}\right)$ of the LHCD grill, and the toroidal mode number $\left(N_{\phi}^{g r}\right)$, or equivalently the toroidal refractive index $\left(n_{\phi}^{g r} \equiv N_{\phi}^{g r} c /\left(\omega R_{m a j}^{g r}\right)\right)$, where $c$ is the speed of light in free space, imposed by the grill as 'boundary conditions.' Shown in Table II are some dimensional and dimensionless characteristic frequencies based upon the plasma properties on the geometrical axis. Maximum values within the plasma volume of some elements of the dielectric tensor are also included in the table. The definitions of these quantities will be given in Sec. III.

Table 2: Parameters of Plasma Models - II

\begin{tabular}{|r|r|r|r|l|}
\hline & TRIAM & PBXM & SUPRA & Units \\
\hline$\omega$ & 5.2 & 4.6 & 2.3 & $10^{10} \mathrm{rad} / \mathrm{sec}$ \\
$\omega_{p e}$ & 2.5 & 3.1 & 3.6 & $10^{11} \mathrm{rad} / \mathrm{sec}$ \\
$\omega_{c e}$ & 12 & 2.6 & 6.9 & $1 r^{-1} \mathrm{rad} / \mathrm{sec}$ \\
$\omega_{L H}$ & 4.1 & 3.3 & 7.4 & $10^{9} \mathrm{rad} / \mathrm{sec}$ \\
$\omega_{g m}$ & 20 & 4.4 & 16 & $10^{9} \mathrm{rad} / \mathrm{sec}$ \\
\hline$\omega_{p e} / \omega$ & 4.9 & 11 & 15 & \\
$\omega_{c e} / \omega$ & 24 & 9.1 & 30 & \\
$\omega_{L H} / \omega$ & 0.08 & 0.11 & 0.32 & \\
$\omega_{g m} / \omega$ & 0.39 & 0.15 & 0.69 & \\
$\left(\omega_{p e} / \omega_{c e}\right)^{2}$ & 0.04 & 1.4 & 0.27 & \\
\hline$|P|$ & 23 & 113 & 235 & \\
$S$ & 1.04 & 2.40 & 1.24 & \\
$|P| / S$ & 22 & 49 & 196 & \\
\hline
\end{tabular}

The pitch angle, $\zeta_{t}$, between magnetic field lines and the toroidal direction will be needed in our analysis. The variation along the midplane of the pitch angle, $\zeta_{t} \equiv \arctan B_{\theta} / B_{\phi}$, where $B_{\theta}$ is the poloidal magnetic field, is plotted in Fig. 1 for all three model plasmas. For the PBXM model plasma, the angle was taken from typical equilibrium calculations. For the TRIAM and SUPRA model plasmas, the angle was calculated from an assumed parabolic radial profile of the safety factor, $q\left(r_{\min }\right)$, with $q(0)=1$. No Shafranov shift was included in these calculations. We note that the pitch angle is larger on the outboard side than on the inboard side for the both circular plasmas (TRIAM and SUPRA), but the angle is comparable on either side for the bean-shaped plasma (PBXM). We note also that the pitch angle is far greater for the SUPRA plasma than for the TRIAM plasma, because the SUPRA model has a much smaller aspect ratio as well as a much smaller $q_{e d g e}$. The pitch angle for the PBXM model plasma is 
enhanced compared to that for a circular plasma (not shown) in the same tokamak because of shaping.

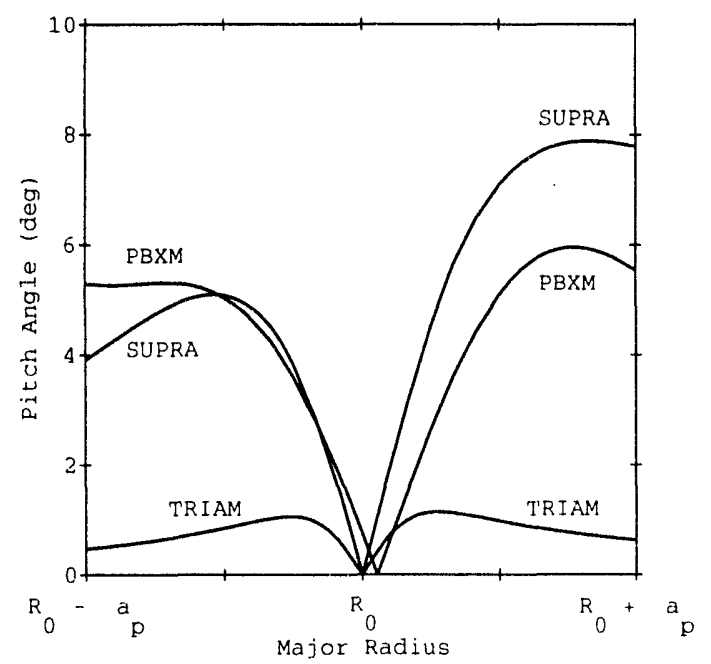

Fig. 1: The variation along the midplane of the magnitude of magnetic field-line pitch angle is shown for the three model plasmas. The angle for the SUPRA model is much greater than that for the TRIAM model, because the SUPRA model has a much smaller aspect ratio as well as a much smaller $q_{\text {edge. }}$. The angle for the PBXM model plasma (bean-shaped) is large compared to that for a similar circular discharge (not shown) because of shaping.

\section{DISPERSION RELATIONS}

We will largely follow the nomenclature used by Stix ${ }^{21,22}$ in revisiting some steps of the development of the theory of lower hybrid wave propagation. The purpose of this section is primarily to introduce the notion of the oblique resonance and to clarify the relationship between the classical and generalized accessibilities. The definitions of terms and symbols, which will be needed in later sections, are also stated.

\section{A. Dielectric Tensor Elements}

Elements of the dielectric tensor for a cold homogeneous plasma, made of electrons and ions of a single species, and immersed in a uniform magnetic field, are defined as,

$$
\begin{aligned}
R & \equiv 1-\frac{\omega_{p e}^{2}+\omega_{p i}^{2}}{\left(\omega-\omega_{c e}\right)\left(\omega+\omega_{c i}\right)}, \\
L & \equiv 1-\frac{\omega_{p e}^{2}+\omega_{p i}^{2}}{\left(\omega+\omega_{c e}\right)\left(\omega-\omega_{c i}\right)}, \\
P & \equiv 1-\frac{\omega_{p e}^{2}+\omega_{p i}^{2}}{\omega^{2}}, \\
S & =\frac{1}{2}(R+L), \\
D & =\frac{1}{2}(R-L),
\end{aligned}
$$


where $\omega_{p s}^{2} \equiv q_{s}^{2} n_{s} /\left(\epsilon_{0} m_{s}\right)$ is the square of the plasma frequency, and $\omega_{s} \equiv q_{s} B / m_{s}$ is the cyclotron frequency, in which the subscript $s$ stands for either the electron (e) or ion (i) species, $\epsilon_{0}$ is the vacuum permittivity, $n_{s}$ is the number density of the species $s, m_{s}$ is the mass of a particle of the species $s$, and $q_{s}$ is the magnitude ${ }^{1}$ of the electronic charge on a particle of the species $s$. The lower hybrid frequency is $1 / \omega_{L H}^{2} \equiv 1 /\left(\omega_{c i}^{2}+\omega_{p i}^{2}\right)+1 / \omega_{g m}^{2}$, where the square of the gm (geometrical mean) gyrofrequency is defined by $\omega_{g m}^{2} \equiv \omega_{c e} \omega_{c i}$. Some of these frequencies are evaluated at the geometrical center of the plasma, and are tabulated in Table I for the three model plasmas. The maximum magnitudes of $P, S$ and $P / S$ encountered within the plasma are also tabulated there.

The full expressions in Eq. 1 are used for all numerical calculations presented in this paper. For analytic evaluations in the current drive regime far away from the lower hybrid resonance condition, approximate expressions valid for $\omega_{c i}^{2} \ll \omega^{2} \ll \omega_{c e}^{2}, \omega^{2} \ll \omega_{p e}^{2}$ and $\omega_{c e} \omega_{c i} \ll \omega^{2}$ are useful, and are given by,

$$
\begin{aligned}
& P=-\frac{\omega_{p e}^{2}}{\omega^{2}}, \\
& S=1+\frac{\omega_{p \epsilon}^{2}}{\omega_{c \epsilon}^{2}}, \\
& D=\frac{\omega_{p e}^{2}}{\omega \omega_{c \epsilon}} .
\end{aligned}
$$

These expressions are valid crer.where in the model plasmas except near their periphery. For conditions close to, or including the lower hybrid resonance, the expression for $S$ should be replaced by $S=1+\left(\omega_{p t}^{\prime} / \omega_{i t}\right)^{2}-\left(\omega_{p i} / \omega^{2}\right)^{2}=1+\left(\omega_{p t} / \omega_{c t}\right)^{2}\left(1-\omega_{c t} \omega_{c i} / \omega^{2}\right)$.

Variations along the midplane of the dielectric tensor elements are shown in Fig. 2 for the PBXM model plasma. Variations along any other plasma diameter are qualitatively similar, and regions of different characteristics referred to in later sections are roughly of either 'ringshaped,' or 'disc-shaped' zones. Variations of the tensor elements for the SUPRA and TRIAM model plasmas (not shown) are qualitatively similar to those for the PBXM plasma, except that the maximum values attained by the individual elements are quite different (see Table I for the maximum values of somc pertinent elements and their ratio).

We note that $S$ is significantly greater than unity for the PBXM model plasma, unlike the other two model plasmas: clectrons are not as 'strongly magnetized' in the PBXM model plasma as they are in the others (see Table I). The plasma density plays a more important role in the PBXM model plasma than in the others through the $\omega_{p e}^{2} / \omega_{c c}^{2}$ term in $S$ (see Eq. 2). We also note that $L<0$ over most of the plasma volume for the PBXM and SUPRA plasmas, but that $L>0$ everywhere for TRIAM plasma. The TRIAM model plasma is in a region of the parameter space that is qualitatively different from that occupied by the others.

It is a feature common to all of these model plasmas that the lower hybrid resonance condition $(S=0)$ is not satisfied anywhere inside the plasma. This feature is in fact a

\footnotetext{
${ }^{1}$ We deviate from Stix's nomenclature and sign convention here: $q_{s}$ is a signed quantity, and $\omega_{e}$ is a negative quantity in his definitions.
} 
characteristic of the lower hybrid current drive regime (in contrast to the lower hybrid ion heating regime): densities are low enough, or frequencies are high enough $\left(\omega_{L H} / \omega \ll 1\right)$, that the lower hybrid resonance does not occur inside the plasma (see Table II).

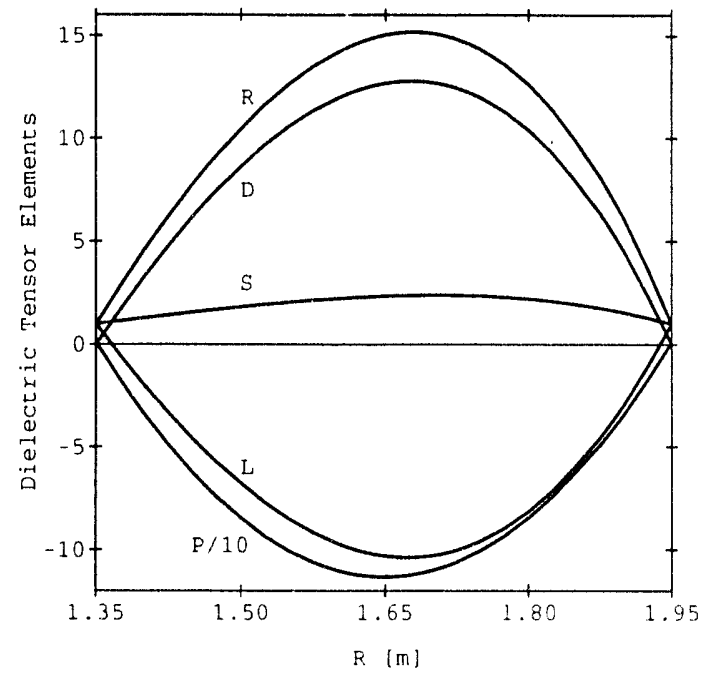

Fig. 2: Variations of cold plasma dielectric tensor elements along the midplane are shown for the PBXM model plasma. The $P$ curve is shown divided by $10 . P=-113$ and $S=2.4$ at their respective maximum magnitude. The value of $S$ deviates significantly from unity, indicating that the electrons are 'weakly magnetized.'

\section{B. Classical Accessibility}

In the framework of usual lower hybrid wave propagation theory, a dispersion relation written for a homogeneous plasma is assumed valid locally at each point in an inhomogeneous plasma. It has been a common practice to solve the dispersion relation for $n_{\perp}{ }^{2}$ for a given value of $n_{\|}$, and present the solution as a function of position (or a plasma parameter, often the density). The presentation is useful for examining how far waves of a given and fixed $n_{\|}$ (i.e., non-upshifting) can penetrate into an inhomogeneous plasma. This is the classical wave accessibility question. A form of the of cold plasma dispersion relation useful for answering the question is given by, ${ }^{21,22}$

$$
\begin{aligned}
a_{4} n_{\perp}{ }^{4} & -a_{2} n_{\perp}{ }^{2}+a_{0}=0, \\
a_{4} & \equiv S, \\
a_{2} & \equiv R L+P S-(P+S) n_{\|}{ }^{2}, \\
a_{0} & \equiv P\left(R L-2 S n_{\|}{ }^{2}+n_{\|}{ }^{4}\right) .
\end{aligned}
$$

Variations along the midplane of the real-valued solutions of Eq. 3 for $n_{\perp}{ }^{2}$ (denoted as $n_{p p}^{2}$ in the figure) are shown in Fig. 3 for a set of $n_{\|}$values for the PBXM model plasma. For the lowest three values, $n_{\|}=2.1,2.45$ and 2.70 , no real-valued solutions are found in a plasma central region. The deepest (smallest 'minor radius') points to which waves propagate are 'turning points' (indicated, e.g., by ' $\mathrm{M}$ ' on the curves for $n_{\|}=2.70$ ). These are points at which the given value of $n_{\|}$satisfies the mode conversion condition. The domain in which real solutions are found is greater on the inboard side on account of a higher magnetic field there. For waves with a sufficiently high $n_{\|}$(e.g., the curves for $n_{\|}=2.75$ ), there are no turning points, and the entire plasma volume is 'accessible' to the waves. 
Diagrams, such as the one in Fig. 3, show how far a wave can penetrate into the plasma. if the wave retains the original $n_{\|}$with which it was launched at the plasma edge. It is also useful to be able to evaluate, through simple calculations, the value of $n_{\|}$that is needed for a wave to penetrate to any given point in an inhomogeneous plasma, or equivalently, the value of $n_{\|}$that satisfies the mode conversion condition at each point in space.

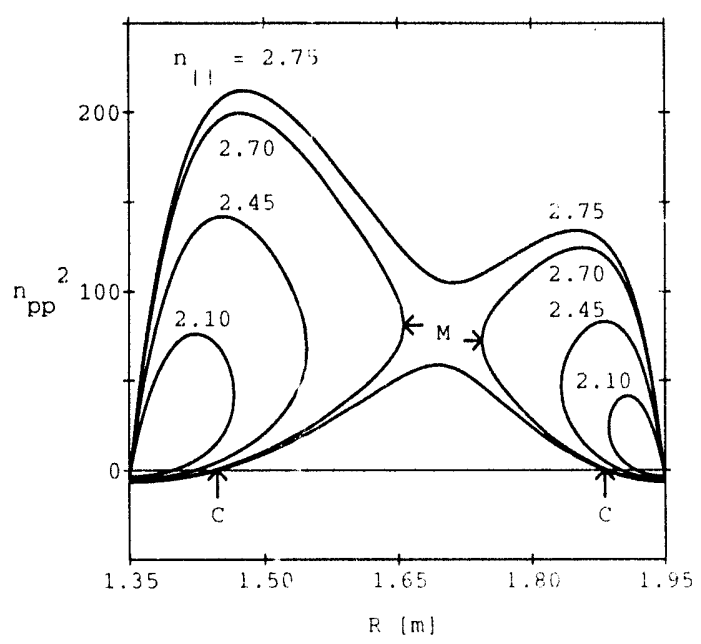

Fig. 3: The classical accessibility of the PBXM model plasma is shown. Only two curves, with $n_{\|}=2.75$ and $n_{\|}=2.70$ (only the one on the inboard side), reach the plasma center. The other curves 'turn around' at a point short of the plasma center. The turning point of a wave (e.g., ' $\mathrm{M}$ ' on $n_{\|}=2.70$ curves) is a point at which the mode conversion condition is satisfied, and the wave changes its character from the lower hybrid wave (the larger $n_{\perp}{ }^{2}$ segment) to the fast wave (the smaller $n_{\perp}{ }^{2}$ segment). The fast wave is cutoff (i.e., $n_{\perp}^{2} \leq$ 0) under some conditions (e.g., "C" on $n_{\|}=$ 2.75 curves).

The condition that solutions to Eq. 3 be real-valued is the mode conversion condition, and is given in its general form by $a_{2}^{2}-4 a_{4} a_{0} \geq 0$. The condition can be re-written in terms of a polynomial in $n_{\|}^{2}$ as,

$$
\begin{aligned}
b_{4} n_{\|}^{4} & -b_{2} n_{\|}^{2}+b_{0}=0, \\
b_{4} & =(P-S)^{2}, \\
b_{2} & =2((R L-P S)(P+S)+2 P S(P-S)) . \\
b_{0} & =(R L-P S)^{2} .
\end{aligned}
$$

This polynomial has two solution branches for $n_{\|}^{2}$ which can be written in terms of the dielectric tensor elements only. Both solutions are complex-valued, and no mode conversion occurs in the thin peripheral region outside the so-called critical layer (see below). In the central plasma region the branch that results in a positive value for $n_{\|}^{2}$ is to be taken. The solution yields a numerical value at each point in space when the tensor elements are evaluated there. We designate this value by $\left(n_{\|}^{M C}\right)^{2}$, and write,

$$
\left(n_{n} \|^{M C}\right)^{2} \equiv \frac{b_{2}+\sqrt{\left(b_{2}^{2}-4 b_{1} b_{0}\right)}}{2 b_{4}} .
$$

Variations of $n_{\|} M C^{\prime}$ along the midplane are shown in Fig. 4 for the three model plasmas. 'The PBXM model plasma represents a comparatively 'inaccessible' situation, while any wave with a $n_{\|}$value only slightly above unity can access the TRIAM model plasma interior.

An approximate expression for $n_{\|}{ }^{M C}$ was derived by Stix. ${ }^{23}$ Except for a thin plasma edge region, conditions, $P<0, L<0$ and $-S / P \ll 1$, hold (see Fig. 2), and $n_{\|}^{M C} \approx$ 
$\sqrt{S}+\sqrt{(} R L / P)$. With an identity, $R L / P \equiv\left(S^{2}-D^{2}\right) / P$, and a further restriction, $S^{2} \ll D^{2}$, an expression identical to Stix's result, $\left.n_{\|}^{M C} \approx \sqrt{S}+\sqrt{(}-D^{2} / P\right)$, is obtained. Noting $-D^{2} / P=S-1$, if the simple expressions for dielectric tensor elements, Eq. 2, are used, a simple mode conversion condition is given by ${ }^{2}$,

$$
n_{\|}^{M C} \approx \sqrt{S}+\sqrt{(S-1)}
$$

Waves with an $n_{\|}$value greater than $n_{\|}{ }^{M C}$ evaluated at a plasma interior point can propagate to that point (under an implicit assumption that simpie monotonic density and magnetic field profiles lead to monotonically increasing $n_{\|}{ }^{M C}$ leading to the point). As $S$ tends to unity, $n_{\|}{ }^{M C}$ also tends to unity. We note that this expression depends only on the parameter, $\left(\omega_{p e} / \omega_{c e}\right)^{2}$. The approximation given by Eq. 6 results in nearly identical values as the exact expression in Eq. 5 for any of the three model plasmas.

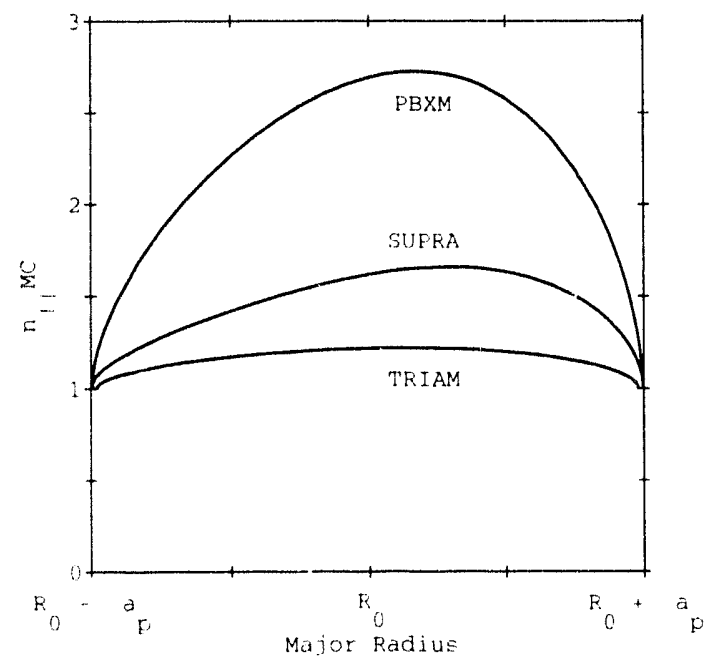

Fig. 4: The variation along the midplane of the value of $n_{\|}$, designated as $n_{\|}{ }^{M C}$, that satisfies the local mode conversion condition, is shown for the three model plasmas. In order for a wave to reach any midplane point in a model plasma, the wave must possess $n_{\|}$equal to, or greater than, $n_{\|}{ }^{M C}$ pertinent to that point.

There may be points on a curve in Fig. 3 at which $n_{\perp}{ }^{2}$ becomes zero (e.g, points indicated by ' $\mathrm{C}$ ' on the curves labeled $n_{\|}=2.75$ ). The condition for this to happen can be found by setting to zero the coefficient, $a_{0}$, defined in Eq. 3. The resulting equation involves only $n_{\|}^{2}$ and dielectric tensor elements. We designate the value of $n_{\|}$that satisfies the equation by $n_{\|} F C$. It is given by,

$$
\left(n_{\|}^{F C}\right)^{2} \equiv R
$$

The point is customarily called a fast wave 'cut-off' point.

A turning point, if one exists, and the plasma edge divide each solution curve in Fig. 3 into two sections: the upper (larger $n_{\perp}{ }^{2}$ ) and lower (smaller $n_{\perp}{ }^{2}$ ) sections are often referred to as the slow waves (or lower hybrid waves) and fast waves, respectively. For waves with large $n_{\|}$, there are no turning points within the plasma, the real-valued solutions are represented by a pair of disconnected curves (see, e.g., curves for $n_{\|}=2.75$ ): the upper curve represents

\footnotetext{
${ }^{2}$ The same result has been obtained by other researchers.
} 
the slow waves and the lower curve represents fast waves. The lower hybrid waves propagate everywhere inside the plasma (except in a very thin layer at the plasma edge-see below), and the fast waves may also propagate in a central region. For waves with still larger $n_{\|}$, the domain of propagation of the fast waves shrinks and finally disappears. It is interesting to note that these 'two' waves, slow and fast waves, discussed here comprise a single branch of the solution to the cold plasma dispersion relation (see below).

It is the variation with $n_{\|}$of the wave penetration depth that is proposed as a tool for controlled modification of the current profile. In addition to the accessibility, however, wave damping must also be considered. Waves may penetrate to a desired location, but may not damp there because of a too low electron temperature. Waves may also damp 'prematurely' before reaching a desired location, if the electron temperature is too high.

\section{Wave Normal Surfaces and Oblique Resonance}

The conventional dispersion relation calculations shown in Fig. 3 are useful for assessing the classical accessibility, and for providing quantitative information on $n_{\perp}$ for a given value of $n_{\|}$. The form of representation is not convenient, however, when the notion of a fixed $n_{\|}$wave is abandoned, and the concept of $n_{\|}$shifting, either upward or downward, is introduced. It also gives little information on the nature of waves. We explore here another form of representation: the dispersion relation is expressed at a given point in space (i.e., for a given set of plasma parameters) in terms of 'wave normal surfaces.'

The cold plasma dispersion relation given in Eq. 3 can be written in terms of the magnitude of normalized phase velocity vector, $u \equiv\left|\vec{u}_{p h}\right|$, where $\vec{u}_{p h} \equiv \vec{k} \omega /\left(k^{2} c\right)$ and $k \equiv|\vec{k}|$, and the zenith angle between the phase velocity and magnetic field vectors, $\zeta_{n}$. The zenith angle is the angle of a vector measured from the local magnetic field vector in the plane that contains both vectors. The phase velocity is normalized by the speed of light in free space. The equation can be written as, ${ }^{21,22}$

$$
\begin{aligned}
A_{0} u^{4} & -A_{2} u^{2}+A_{4}=0, \\
A_{4} & \equiv S \cos ^{2} \zeta_{n}+P \sin ^{2} \zeta_{n}, \\
A_{2} & \equiv R L \sin ^{2} \zeta_{n}+P S\left(1+\cos ^{2} \zeta_{n}\right), \\
A_{0} & \equiv P R L .
\end{aligned}
$$

Solutions to this equation can be represented in terms of wave normal surfaces.

A wave normal surface is the locus traced by the tip of the normalized wave phase velocity vector as the wave propagation zenith angle is varied. A wave normal surface is a threedimensional (3D) surface of revolution about its vertical axis, which is the parallel component, $u_{\|}$, of the normalized phase velocity vector ${ }^{3}$, and is symmetric about the plane, $u_{\|}=0$. 'Two

\footnotetext{
${ }^{3}$ Following a perhaps unfortunate but widely-accepted convention, we earlier used the term 'parallel phase speed,' to mean the quantity, $\omega / k_{\|}$, where $k_{\|}$is the parallel component of the wave vector, $\vec{k}$, rather than the parallel component of the phase velocity vector, $\omega / \vec{k}$, as defined here. The latter quantity is $\omega k_{\|} / k^{2}$
} 
types of the wave normal surface are encountered in the LHCD field, and are the spheroid and 'dumbbel' lemniscoid (or a rotated 'figure eight'). A lemniscoid wave normal surface is depicted in Fig. 5. Only the upper half $\left(u_{\|} \geq 0\right)$ of the rotated 'figure eight' is shown in its vertical cross section. The topological genus of wave normal surfaces have been used to classify the type of solutions to the cold plasma dispersion relation in the Clemmow-Mullaly-Allis (CMA) diagram. In the diagram the parameter space is divided by 'bounding surfaces' into various 'bounded volumes' in which the wave normal surfaces remain topologically the same. The topological characteristics obey a set of well-defined rules $^{22}$; for example, the two surfaces never intersect each other, and if they touch each other, they do so only either at the 'north and south poles' or along the 'equator' of the surfaces. Classifications of wave types based upon the topology of wave normal surfaces are less prone to confusion caused by semantics (such as 'fast' or 'slow' waves).

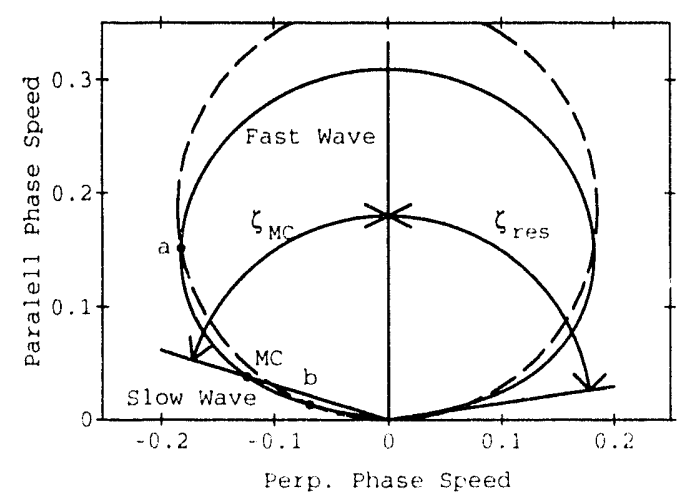

Fig. 5: The lemniscoid wave normal surface (upper half only) is shown by a solid curve for a mid-radius point on the inboard side ( $R=1.5 \mathrm{~m}, z=0 \mathrm{~m}$ ) for the PBXM model plasma. A lemniscoid wave normal surface possesses a resonance at an oblique angle, $\zeta_{\text {res }}$. The part of the surface near the resonance, up to the mode conversion point (MC) is usually referred to as 'slow waves,' and the remaining part as 'fast waves.' The locus of constant $n_{\|}=2.7$ is shown in dashed line. The intersections of the solid curve and dashed line are the 'two' solutions shown in Fig. 3.

Variations of the dielectric tensor elements near the plasma edge on the inboard (small $R_{m a j}$ ) side are shown in Fig. 6 for the PBXM model plasma (a greatly magnified edge region of Fig. 2). Each horizontal division represents $1 \mathrm{~cm}$. Conditions, $P=0$ and $L=0$ (each indicated by a short vertical dashed line), divide the plasma into three regions labeled, ' $6 \mathrm{a}$,' '7,' and ' $8 \mathrm{a}$.' These conditions are two of the bounding surfaces of the CMA diagram. The labels used here correspond to those used by $S_{t i x}{ }^{21,22}$ in identifying bounded volumes of the CMA diagram (see Figs. 2-1 and 2-2 of the references cited). Associated with each bounded volume there are up to two wave normal surfaces, which are sketched in the insets of Fig. 6 (without the scale to indicate their size). Each curve in the insets represents a 2-D vertical cross section of a 3D figure of rotation about the vertical axis. The shape of a curve in detail may change (e.g., from circle to ellipse), but its topological characteristics remains unchanged at different points within each bounded volume.

We note $P>0$ within a very thin (ring) region, '6a,' at the plasma edge. The region is only $1.3 \mathrm{~mm}$ in width on either the inboard or outboard side of the PBXM model plasma. The corresponding number is $1.7 \mathrm{~mm}$ for the SUPRA model plasma, and $2.5 \mathrm{~mm}$ for the TRIAM model plasma. Each of the two branches of the dispersion relation has a wave normal surface that is topologically a spheroid. The surfaces shown in Inset (a) of Fig. 6 are for a point $1 \mathrm{~mm}$ from the inboard edge of the plasma $\left(R_{m a j}=1.351 \mathrm{~m}\right)$. The outer wave normal surface ('faster' 
wave) has a left-handed polarization (L) for parallel propagation and an ordinary polarization (O) for perpendicular propagation. The inner wave normal surface ('slower' wave) has a righthanded $(\mathrm{R})$ polarization for parallel propagation, and an extraordinary $(\mathrm{X})$ polarization for perpendicular propagation. A spheroid surface (not shown) corresponding to the speed of light in free space would intervene between the two surfaces, but lie very close to the inner surface. At the plasma edge (in vacuum), the two surfaces become identical, and at the bounding surface, $P=0$, they touch each other at the 'north and south poles.' The surface is often called the 'critical layer.'

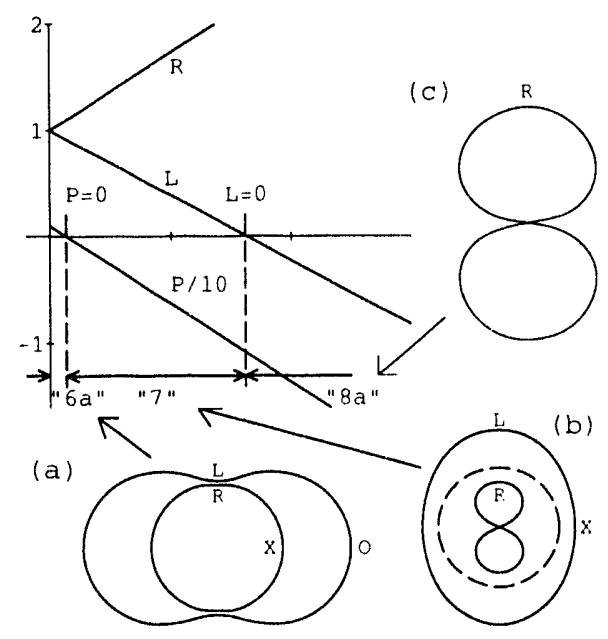

Fig. 6: The region near the inboard edge of the PBXM model plasma is divided into three 'bounded volumes,' designated as ' $6 \mathrm{a}$, ' ' 7 ' and '8a,' by 'bounding surfaces,' $P=0$ and $L=0$, of the CMA diagram. In each bounded volume the topology of wave normal surfaces is shown: the volume, ' $6 \mathrm{a}$,' has two solution branches, each with a spheroid wave normal surface, the volume, ' 7 , has two branches, one with a spheroid surface and the other with a lemniscoid surface. The volume, ' $8 \mathrm{a}$,' has only one branch with a lemniscoid surface. In the insets, letters, 'L,' 'R,' 'O,' and ' $\mathrm{X}$ ' refer to the polarization of the wave electric field.

Within the next thin region, ' 7 ,' at the plasma edge (as well as in ' $6 \mathrm{a}$ '), we note $L>0$. The region is $1.6 \mathrm{~cm}$ in width on the inboard side, and $1.2 \mathrm{~cm}$ on the outboard side, for the PBXM model plasma. The corresponding numbers are more substantial for the SUPRA model plasma, and are $7.5 \mathrm{~cm}$ and $4.0 \mathrm{~cm}$, respectively. For the TRIAM model plasma, $L>0$ everywhere, and the entire plasma interior belongs to the region, ' $T$.' One solution in this region, labeled ' $\mathrm{L}-\mathrm{X}$,' still has a spheroid wave normal surface (' $\mathrm{O}$ ' and ' $\mathrm{X}$ ' labels were interchanged at the critical layer), and the other has a lemniscoid surface. 'The surfaces shown in Inset (b) of Fig. 6 are for a point $1 \mathrm{~cm}$ from the inboard edge of the plasma $\left(R_{m a j}=1.36 \mathrm{~m}\right)$. A spheroid surface corresponding to the speed of light in free space intervenes between the two surfaces, and is shown by a dashed curve. The curve helps to gauge the phase speed: the spheroid and lemniscoid branches represent waves travelling faster $(u>1)$ or slower $(u<1)$ than the speed of light, respectively. At the bounding surface, $L=0$, which is a cut-off, the L-X wave normal surface disappears. Within the remaining central region, '8a,' $P<0$ and $L<0$. Note that this region occupies most of the plasma volume for the PBXM and SUPRA model plasmas. It is interesting to note that there is only one branch, which has a lemniscoid wave normal surface, to the solution of the dispersion relation in this region.

Except in the very thin region, ' $6 \mathrm{a}$,' at the plasma edge, waves concerned in the conventional LHCD theory are everywhere characterized by a lemniscoid wave normal surface. An example of the lemniscoid wave normal surface in the bounded volume, ' $8 \mathrm{a}$,' was shown earlier in Fig. 5. 
A lemniscoid surface has a resonance at an oblique nenith angle, $\zeta_{\text {res }}<\pi / 2$, and the phase speed tends to zero there (or, equivalently, both $n_{\|}$and $n_{\perp}$ tend to infinity). The surface shown here (and also in Inset (c) of Fig. 6) is for a mid-radius point on the inboard side $(R=1.5 \mathrm{~m}$, $z=0 \mathrm{~m}$ ) in the PBXM model plasma, and the resonant angle is $\zeta_{\text {res }}=81.6 \mathrm{deg}$. The $\mathrm{R}$ wave normal surface in the bounded volume, '7,' in Inset (b) of Fig. 6 is a lemniscoid, but has a much smaller resonance angle $\left(\zeta_{\text {res }}=68 \mathrm{deg}\right.$ at $1 \mathrm{~cm}$ from the plasma edge for the PBXM model plasma), than in the ' $8 \mathrm{a}$ ' volume.

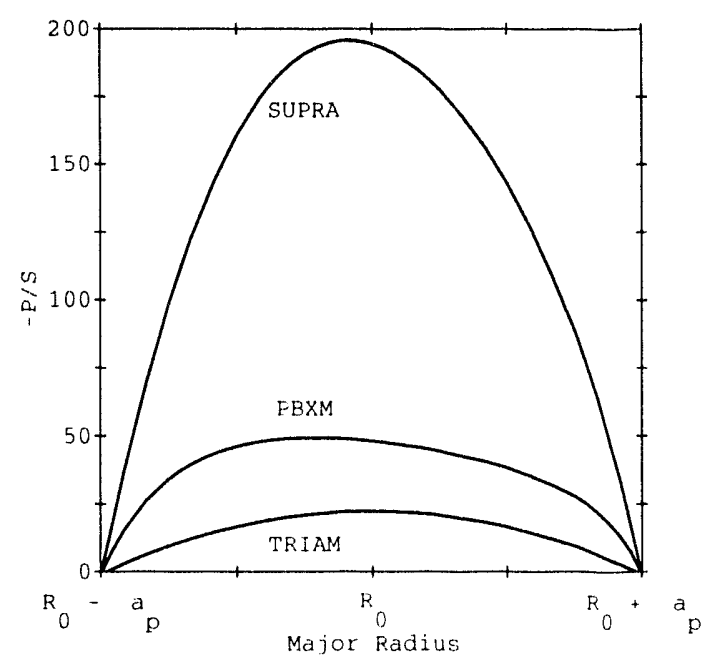

Fig. 7: The variation of a dimensionless 'density,' $-P / S$, along the midplane is shown for the three model plasmas. The PBXM and TRIAM plasmas are low 'density' plasmas, and the SUPRA plasma is a high 'density' plasma. Low 'density' plasmas have a small oblique resonance angle, and high 'density' plasma have a large resonance angle.

In the bounded volume, ' 6 a,' the classical accessibility representation shown in Fig. 3 has two solutions with a purely imaginary $n_{\perp}$ value, and has no counter-parts among wave normal surfaces shown in Fig. 6. This is a consequence of the choice made in Fig. 3 of a fixed $n_{\|}$that is significantly greater than unity. In the bounded volume, ' 7 ,' the classical representation has a solution with a real $n_{\perp}$ value, which corresponds to the lemniscoid solution, and another solution with a purely imaginary $n_{\perp}$ value, which has no counter-part wave normal surface. In the bounded volume, ' $8 \mathrm{a}$,' the classical representation has two solutions with a real-valued $n_{\perp}$, which corresponds to the single lemniscoid wave normal surface there. It may seem peculiar that the 'two' solutions belong to the same wave normal surface. The apparent conflict can be resolved by plotting the trajectory of constant $n_{\|}$together with the wave normal surface. The normalized phase velocity and refractive index vectors have a vector reciprocal relationship, i.e., $\vec{u}=(\vec{n})^{-1}$ : in terms of vector components, $u_{\|}=n_{\|} /\left(n_{\|}{ }^{2}+n_{\perp}{ }^{2}\right)$ and $u_{\perp}=n_{\perp} /\left(n_{\|}{ }^{2}+n_{\perp}{ }^{2}\right)$. The trajectory is represented by two spheres tangent to each other at the origin, and is shown by a dashed curve in Fig. 5 (only the upper sphere is shown in its vertical cross section). The intersections of the solid curve (wave normal surface) and dashed trajectory are the 'two' solutions shown in Fig. 3: the intersection with a greater value of $u$ is the fast wave and the one with a smaller value of $u$ is the slow wave. At the turning point (in space) for the chosen value of $n_{\|}$, the dashed trajectory becomes tangent to the wave normal surface at the mode conversion point (in $\vec{u}$ space) designated by 'MC' in Fig. 5, and the two solutions coalesce. The wave propagation angle at this point is designated as $\zeta_{M C}$ in the figure.

There are two types of propagating waves ( $\mathrm{R}-\mathrm{X}$ and $\mathrm{L}-\mathrm{O}$ ) in the bounded volume, ' $6 \mathrm{a}$,' 
and one type (L-X) in the volume, '7,' that are absent in the classical representation of the accessibility. Roles played by these waves, if any, have been little explored in the conventional LHCD theory. These waves have phase speeds comparable to (and slightly below), or greater than the speed of light in free space, and will interact little with electrons. They can, however, carry energy away into plasma peripheral regions as 'surface waves,' and can be important from a power balance point of view. In early LHCD experiments, grills consisting of only a few large waveguides were used, and the $n_{\|}$spectrum of electric field generated by the waveguides ('waveguide' spectrum) extended to values well below unity. Modern grills with a large number of small waveguides have a sharply defined peak in the waveguide spectrum centered around a desired $n_{\|}$value. But the overall size of the grill (rather than the width of individual waveguides) defines another characteristic scale length that must be represented by a spectrum of low $n_{\|}$values. Even the modern grills thus have a waveguide spectrum extending to small $n_{\|}$values. The so-called Brambilla spectrum ${ }^{24}$ refers only to that part of the waveguide power spectrum that is carried away by propagating waves having $n_{\|}>1$. The waves, missing in the classical accessibility representation, do not therefore manifest themselves in the Brambilla spectrum. (Those waves, that are considered in the conventional theory, but have small grill-launched $n_{\|}$(see, for example, $n_{\|}=2.1$ curve in Fig. 3 ) and thus have poor accessibility, remain in the plasma periphery, and may also be regarded as a form of 'surface waves.' The surface waves discussed in the present paragraph are, however, distinct from these poor accessibility waves.) Diagrams used extensively in the classical accessibility analysis, such as the one shown in Fig. 3, appear to depict 'two' waves, and thus give a false impression that the complete solutions to the bi-quadratic dispersion relation are represented.

The dispersion relation in polar form, Eq. 8, readily yields the condition for the oblique resonance. By setting $A_{4}=0$, and thereby allowing one solution for $n_{\|}$to tend to infinity, we obtain,

$$
\tan ^{2} \zeta_{\text {res }}=-\frac{P}{S}
$$

This resonance ${ }^{4}$ was first described by Allis $^{25}$ (see also Stix ${ }^{21,22}$ ). The right-hand side (RHS) of this equation, which determines the resonance angle, can be regarded as a dimensionless 'density,' and is plotted in Fig. 7 for all three model plasmas. The PBXM model plasma has significantly lower dimensionless densities than the SUPRA model plasma. An examination of the numbers in Table II and Fig. 2, shows that these low dimensionless densities are in part a result of high values of $S$. High $S$ values are in turn a result of high ratio, $\left(\omega_{p e} / \omega_{c e}\right)^{2}$ (see Eq. 2). The oblique resonance angle, $\zeta_{\text {res }}$, is plotted in Fig. 8 for all three model plasmas. The deviation of the resonance angle from a right angle $(\pi / 2)$ is an important factor, together with the geometrical constraint angle (see below), in determining the nature of wave propagation. The deviation is more than three times greater for the SUPRA model plasma than for the TRIAM model plasma.

\footnotetext{
${ }^{4}$ It is curious to note that the singular nature of the dispersion relation under this condition has been recognized in subsequent publications by many authors, it is seldom referred to as a 'resonance.'
} 


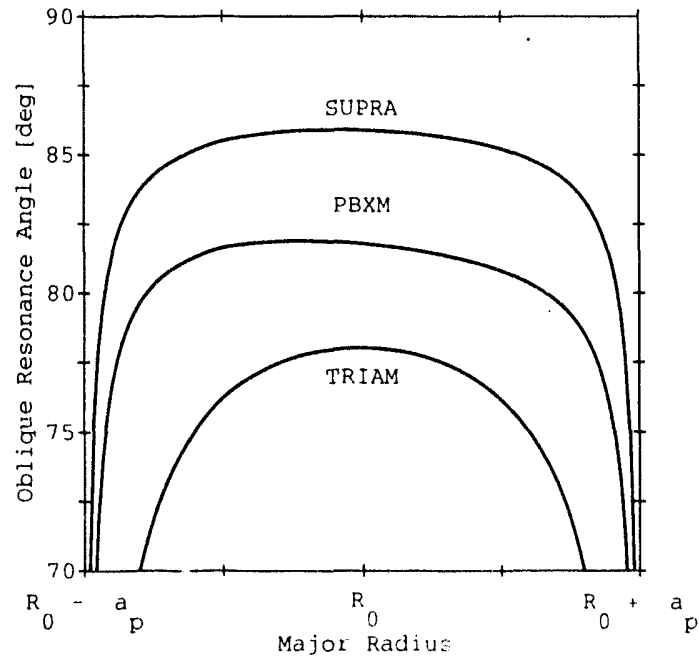

\section{Inverse Wave Normal Surfaces and Electrostatic Approxima- tion}

The dispersion relation can also be expressed in terms of the magnitude and zenith angle of the refractive index vector. $n \equiv|\vec{n}|$ and $\zeta_{n}$, respectively. This 'polar' form of the equation is written as,

$$
A_{4} n^{4}-A_{2} n^{2}+A_{0}=0 .
$$

This equation, governing the refractive index, $n$, is a 'reciprocal' of the earlier equation, Eq. 8 , governing the normalized phase speed, $u$, where the coefficients, $A_{0}$ through $A_{4}$, were also defined.

The solution to the full fourth degree dispersion relation, Eq. 10, at a mid-radius point on the inboard mid-plane, $R_{m a j}=1.5 \mathrm{~m}$ and $Z=0 \mathrm{~m}$, of the PBXM model plasma, is shown in Fig. 9 (top inset) in the form of three-dimensional (3D) surfaces in the refractive index space, $\left(n_{x}, n_{y}, n_{z}\right)$. The solution surfaces, which extend to $\pm \infty$, are a figure of revolution about the $n_{z}$ axis. The axis is aligned with the direction of the local magnetic field line, i.e., $n_{\|} \equiv n_{z}$, and its scale is exaggerated five-fold for clarity of presentation. The surfaces are also symmetric about the $n_{z}=0$ plane. Surfaces, such as these, are the 'reciprocal' of wave normal surfaces, and are sometimes called 'inverse wave normal surfaces.' The 'bottom' of the upper surface (or the 'top' of the lower surface) is at the values of $n_{\perp}$ for which the mode conversion condition (see Eq. 5) is satisfied for the set of plasma parameters at this spatial location. The solution in the vicinity of the mode conversion will be discussed in more detail in a later section. The solution surfaces at large values of $n_{\perp}$ (or $n_{\|}$) asymptotically approach a pair of conical surfaces that are joined at the vertex. These asymptotic cones are shown in Fig. 9 (bottom inset).

An approximate form of the dispersion relation, known as the 'electrostatic approximation,' is used frequently in lower hybrid theories, and is given by,

$$
S n_{\perp}^{2}+P n_{\|}^{2}=0 \text {. }
$$


This is an equation that describes the asymptotic cones of the full solution surfaces. The vertex half-angle of either cone equals the oblique resonance angle at this spatial location. The oblique resonances of a lemniscoid wave normal surface thus correspond to the asymptotic cones of an inverse wave normal surface. The cones do not represent the solution for small values of $n_{\perp}\left(\right.$ or $\left.n_{\|}\right)$.
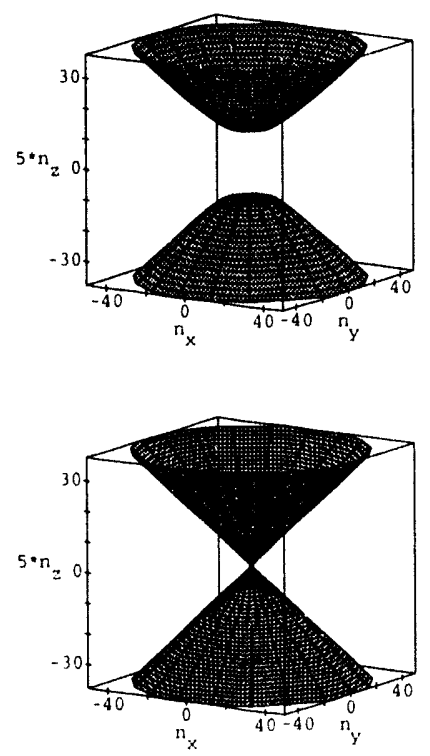

Fig. 9 Solutions to the full fourth degree (upper picture) and E.A. (lower picture) dispersion relations are shown in the refractive index space, $\left(n_{x}, n_{y}, n_{z}\right)$. The surfaces, which extend to $\pm \infty$, are a figure of revolution about the $n_{z}$ axis. The axis is aligned with the direction of local magnetic field line, i.e., $n_{\|} \equiv n_{z}$, and its scale is exaggerated five-fold for clarity of presentation. The surfaces are also symmetric about the $n_{z}=0$ plane. A 'gap' in the middle of the full solution is caused by mode conversion.

The solutions to a homogeneous cold plasma dispersion relation are represented as surfaces of revolution about the local magnetic field line, and have no preferred azimuthal angles about the field line. The dispersion relation can be well represented in two dimensions without a loss of information. But directional preferences may arise through other reasons: for example, a ray tracing analysis introduces the direction of the inhomogeneity of a parameter into the problem. In the present analysis, it will be a constraint imposed by the requirement of the invariance of toroidal mode number that introduces a directional preference.

\section{GEOMETRICAL CONSTRAINT RELATION}

It is usually assumed in LHCD analyses in the toroidal geometry that the plasma is axisymmetric: there are no variations in zero order parameters in the toroidal direction. An immediate consequence of the toroidal symmetry assumption is that the toroidal mode number, i.e., the number of whole wave periods around the torus, is invariant as waves propagate through the plasma. This assumption of the toroidal mode number invariance is commonly adopted in ray tracing analyses.

The wavelength must become shorter in regions of a smaller major radius in order to accommodate the same number of wave periods within a shorter toroidal circumference. The toroidal mode number, $N_{\phi}^{g r}$, imposed by the grill located at $R_{m a j}^{g r}$, is related to the toroidal component, $n_{\phi}^{g r}$, of the refractive index vector at the grill, through $n_{\phi}^{g r}=c N_{\phi}^{g r} /\left(\omega R_{m a j}^{g r}\right)$. 
The constancy of the mode number $\left(N_{\phi}=N_{\phi}^{g r}\right)$ then requires the toroidal refractive index, $n_{\phi}=c N_{\phi} /\left(\omega R_{m a j}\right)$, to be inversely proportional to the major radius, i.e.,

$$
n_{\phi}=\frac{R_{m a j}^{g r}}{R_{m a j}} n_{\phi}^{g r} .
$$

This is a most basic toroidal effect, and will be called a 'wedge effect' for short in this article in reference to the ever narrower width of a wedge toward its apex.

A greater toroidal effect is the geometrical constraint relationship between $n_{\|}$and $n_{\perp}$ arising from the same invariance requirement. We designate a unit vector in the toroidal direction by $\hat{\phi}$, and the magnetic field vector by $\vec{B} \equiv B \hat{b}$, where $B$ is the magnitude of the field, and $\hat{b}$ is a unit vector in the direction of $\vec{B}$. Geometrical relationships among $\hat{\phi}, \hat{b}$, and $\vec{n}$ are shown in Fig. 10. We consider a point, $O$, on a magnetic flux surface, and a local right-handed coordinate system, $(x, y, z)$, about the point. The $x$-axis is perpendicular to the flux surface, and points into the volume enclosed by the flux surface. The $y$ - and z-axes are in the plane tangent to the flux surface ('tangent plane'). The $z$-axis is aligned with the local magnetic field direction. The orientation of a vector with respect to $\hat{b}$ is defined by a set of azimuthal and zenith angles. The azimuthal angle, $\chi$, is the angle that a plane between the vector of interest and $\vec{b}$ makes with respect to the quarter plane defined by the positive $\mathrm{x}$ and $\mathrm{z}$ axes, and ranges over $-\pi<\chi \leq \pi$. A positive azimuthal angle is measured from the positive $x$-axis toward the positive $y$-axis, and a negative azimuthal angle is measured from the positive $x$-axis toward the negative $\mathrm{y}$-axis. The zenith angle, $\zeta$, is the angle that the vector of interest makes with respect to $\hat{b}$ in the plane containing both vectors, and ranges over $0 \leq \zeta \leq \pi$. Note that the term, 'azimuthal,' and the associated symbol, $>$, are used here to niean the angle about a local magnetic field line. It should not be confused with a commonly used term, 'azimuthal' angle, of a spatial point in the plasma poloidal cross section. (We will use the term, 'poloidal' angle for the coordinate of a spatial point.)

The refractive index vector, $\vec{n}$, can thus be designated by $\left(n_{x}, n_{y}, n_{z}\right)$, or by $\left(n, \zeta_{n}, \lambda_{n}\right)$. Waves propagate inward when $n_{x}>0$, or $-\pi / 2<\lambda_{n}<\pi / 2$. The toroidal unit vector, $\hat{\phi}$, can be designated by $\left(0, \hat{\phi}_{y}, \hat{\phi}_{z}\right)$, or by $\left(1, \zeta_{t}, \lambda_{t}\right)$. From the definition of the local coordinate system chosen, $\backslash_{t}$ is either $+\pi / 2$ or $-\pi / 2$, depending upon whether $\hat{\phi}$ lies on the positive or negative $y$-axis side of $\hat{b}$, respectively. The relative location of these two unit vectors is in turn determined by the relative sense of the toroidal magnetic field and plasma current. It can be shown that when the toroidal field and plasma current are 'co-parallel,' $\chi_{t}=+\pi / 2$, and when they are 'counter-parallel,' $\chi_{t}=-\pi / 2$. The statement applies regardless of the spatial location considered. Under a usual current drive situation, the plasma current is assisted by an LHCD driven current (or the entire plasma current is an LHCD driven current). Electrons are expected to be transported by waves in the direction opposite from that of the plasma current under these circumstances. The wave spectrum imposed by the grill must have an appropriate sign: $n_{\phi}^{g r}<0$ for the co-parallel current, and $n_{\phi}^{g r}>0$ for the counter-parallel current. Unless toroidal reflection of waves occurs, $n_{\|}$must have the same sign as $n_{\phi}^{g r}$ everywhere inside the plasma. For the co-parallel current, $\pi / 2<\zeta_{n} \leq \pi$, and for the counter-parallel current, $0 \leq \zeta_{n}<\pi / 2$. The vectors shown in Fig. 10 depict a wave propagating inward in a tokamak with a counter-parallel current. 
In Fig. 10 the toroidal refractive index, $n_{\phi}$, consists of two contributions, one involving $n_{\|}$ and the other involving $n_{\perp}$,

$$
n_{\phi}=n_{\|} \cos \zeta_{t}+n_{\perp} \sin \chi_{n} \sin \chi_{t} \sin \zeta_{t} .
$$

We note that $n_{\|}$may be either positive or negative in general, but $n_{\perp}$ is either positive or zero by the convention adopted here. This equation states that, if $n_{\|}$is to become arbitrarily large, while holding $n_{\phi}$ finite, $n_{\|}$and $n_{\perp}$ must both tend to infinity, and make contributions to $n_{\phi}$ having the opposite signs. Only an oblique resonance can satisfy these requirements. The magnetic field-line pitch angle is $\zeta_{t} \ll \pi / 2$, and $\sin \zeta_{t} \geq 0$ and $\cos \zeta_{t} \geq 0$. We substitute $\sin \chi_{t}= \pm 1$, and solve the above equation for $n_{\|}$to obtain,

$$
n_{\|}=\frac{n_{\phi}}{\cos \zeta_{t}} \pm n_{\perp} \sin \chi_{n} \tan \zeta_{t}
$$

where the plus sign is for the counter-parallel plasma current, and the minus sign is for the co-parallel plasma current. We will call this constraint relationship arising out of the magnetic geometry of the tokamak as 'geometrical constraint.' The term will be abbreviated as 'G.C.'

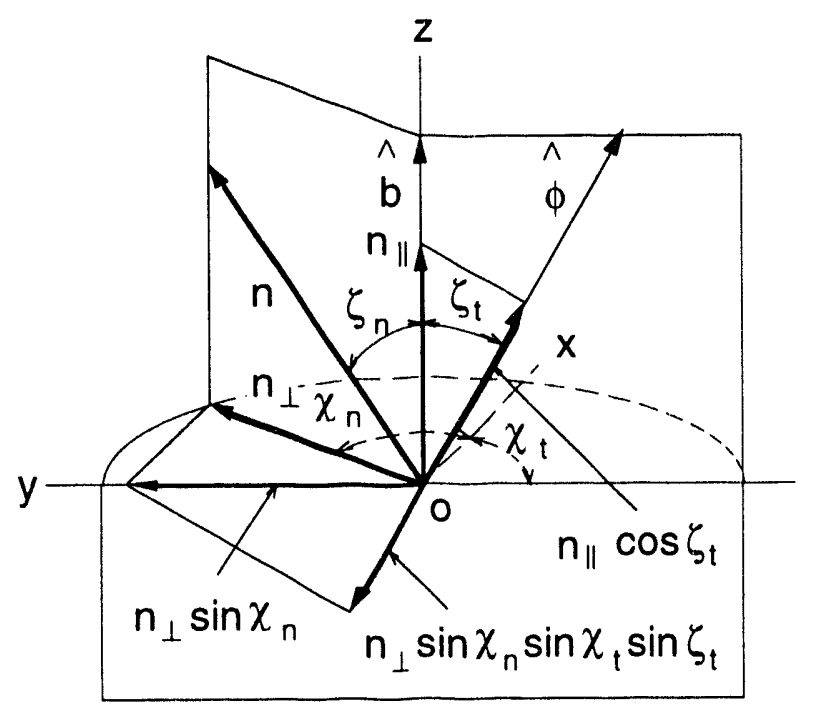

Fig. 10: Geometrical relationships among the refractive index vector, $\vec{n}$, unit vectors in the magnetic field direction, $\dot{b}$, and in the toroidal direction, $\dot{\phi}$, are shown graphically. The magnetic surface is in the plane of the page, and waves propagating inward are depicted.

Noting that $n_{\|} \equiv n_{z}$ and $n_{\perp} \sin \zeta_{n} \equiv n_{y}$, the above equation can also be written as, $n_{z}=n_{\phi} / \cos \zeta_{t} \pm n_{y} \tan \zeta_{t}$. This is a linear relationship between $n_{z}$ and $n_{y}$, independent of $n_{x}$, and can thus be represented as a plane in the $\vec{n}$-space tilted by an angle, $\alpha_{g c}$. Since $\tan \alpha_{g c}=d n_{z} / d n_{y}=\tan \zeta_{t}$, the tilt angle from the horizontal in the $\vec{n}$-space is simply the magnetic field line pitch angle in the physical space, $\alpha_{g c}=\zeta_{t}$. We also define the degree of tilt measured from the vertical by the zenith angle that the plane makes with respect to the vertical axis $\left(n_{z}\right.$-axis) in the $n_{y}-n_{z}$ cross-sectional plane,

$$
\zeta_{g e o}=\pi / 2-\zeta_{t}
$$

The G.C. plane intersects the $n_{\|}$axis at,

$$
n_{\|}^{u d g} \equiv \frac{n_{\phi}}{\cos \zeta_{t}}
$$

where $n_{\phi}$ is given by Eq. 12 . The $n_{\|^{-}}$axis intercept represents the 'wedge effect.' 


\section{GENERALIZED ACCESSIBILITY}

\section{A. Geometrical Interpretation of Admissible Solutions}

The dispersion relation and G.C. relation must be satisfied simultaneously by $n, \zeta_{n}$, and $\chi_{n}$ at every spatial point. In the present analytical treatment, we will leave one of the three parameters indeterminate, and obtain a range of solutions that is 'admissible.' Ray tracing calculations can determine not only all three parameters, but also the power carried by the wave. Nevertheless, solutions that are not allowed in the present analysis cannot be solutions of a ray tracing calculation, because both analyses are founded on the same set of assumptions. Many qualitative observations useful in interpreting results from ray tracing calculations and experiments can be made from an examination of admissible solutions.

In Fig. 11, the E.A. dispersion relation and G.C. relation are represented by two surfaces in a $3 \mathrm{D} \vec{n}$-space. Only the upper half $\left(n_{z} \equiv n_{\|} \geq 0\right)$ of the space is shown. The intersection of the surfaces represents simultaneous solutions to the E.A. and G.C. relations. A case without a poloidal field (zero field-line pitch angle) is considered here. The G.C. plane is therefore horizontal, and the intersection is a circle. There are no preferred azimuthal directions of propagation in this case. Lower hybrid wave propagation in the classical accessibility can also be described by these surfaces.

The same set of surfaces is shown in Fig. 12 for a case with a poloidal field (finite fieldline pitch angle). Both pitch angle and oblique resonance angle are modest in this plasma. Consequently, the tilt of the C.C. plane off the horizontal is weak, and the E.A. cone has a narrow vertex angle. Under these circumstances, the oblique resonance zenith angle is inside the G.C. zenith angle $\left(\zeta_{\text {res }}<\zeta_{g e o}\right)$, and the oblique resonance is not an admissible solution. The G.C. plane also intersects only the upper E.A. cone.

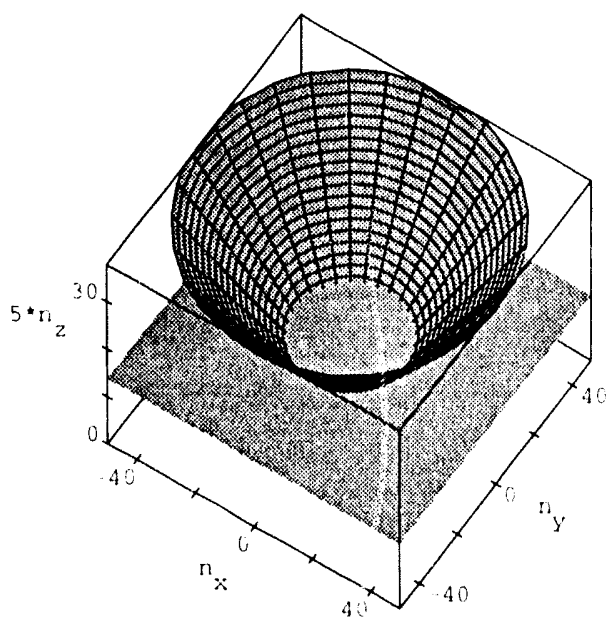

Fig. 11 The upper E.A. cone and G.C. plane are shown: the cone is for a mid-radius point on the inboard midplane $\left(R_{m a j}=1.50 \mathrm{~m}\right.$ and $\left.z=0 \mathrm{~m}\right)$ of the PBXM model plasma, and the G.C. plane is for a case without a poloidal field (zero field-line pitch angle). A wave with $n_{\phi}=2.1$ is assumed to be launched from the plasma edge in the outboard midplane. The G.C. plane is horizontal, and the intersection of these surfaces is a circle.

The intersection of the E.A. cone and G.C. plane is a planar space curve, which is an ellipse in its own plane. The space curve is presented in the same 3D $\vec{n}$-space in Fig. 13 . A vector 
drawn from the origin to any point on the curve represents an admissible solution. A few such vectors are indicated by solid and broken lines in the figure. Projections of the solution space curve and vectors onto (a) $n_{x}-n_{z}$ plane, (b) $n_{y}-n_{z}$ plane, and (c) $n_{x}-n_{y}$ plane are shown in Fig. 14.
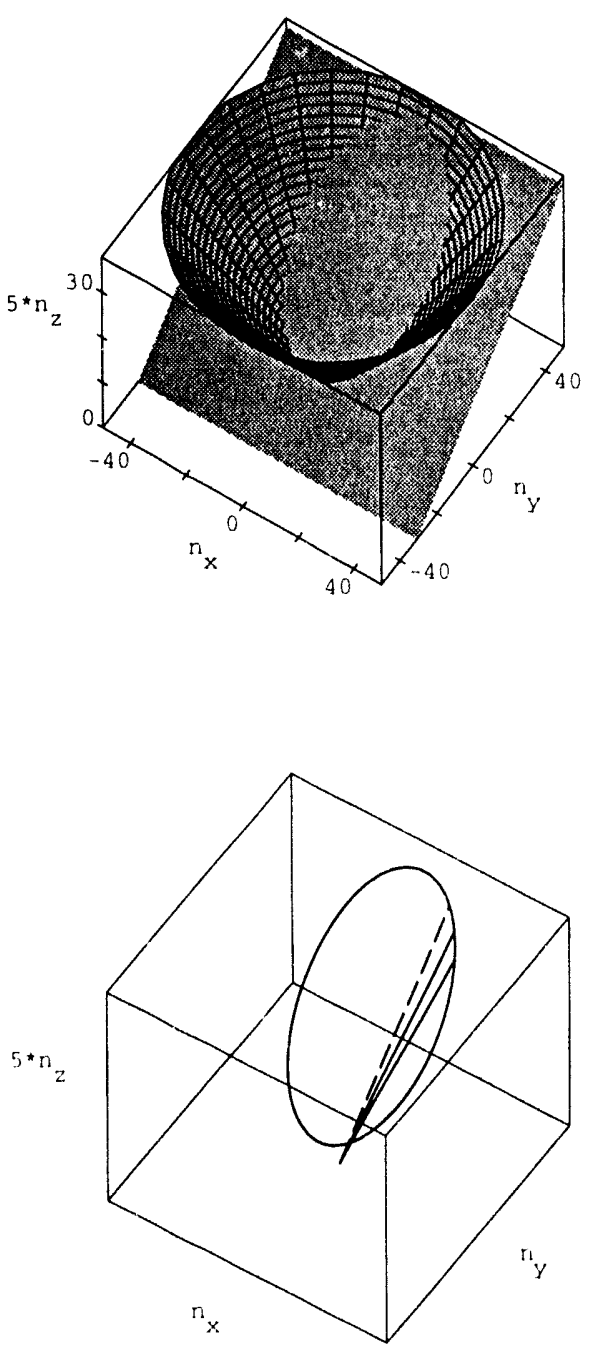

Fig. 12 The upper E.A. cone and G.C. plane are shown: the both are for a mid-radius point on the inboard midplane $\left(R_{m a j}=1.50 \mathrm{~m}\right.$ and $\left.z=0 \mathrm{~m}\right)$ of the PBXM model plasma. The G.C. plane now represents a case with a poloidal field (finite fieldline pitch angle). The toroidal magnetic field is in the positive toroidal direction, and the plasma current is counter-parallel to the field. A wave with a positive $n_{\|}$value $\left(n_{\phi}=2.1\right)$ is assumed to be launched from the plasma edge in the outboard midplane. The G.C. plane is therefore tilted upward toward the positive $n_{y}$ direction (a choice of the plus sign in Eq. 14). The intersection of the surfaces is an ellipse.

Fig. 13 The intersection of the upper E.A. cone and G.C. plane is shown for the same conditions as in Fig. 12. The solution curve is an ellipse in its own tilted plane. A few vectors drawn from the origin to points on the curve are indicated by solid and broken lines. The origin of the vector space is located on the bottom of the 'box.' These vectors represent admissible solutions.

An examination of the solution space curve reveals a number of qualitative but useful characteristics of the solution at this spatial location. The solution curve is in a tilted plane, and $n_{\|}$of admissible solutions is no longer a single value as it was in Fig. 11 . The $n_{\|}$value of the actual solution depends on the wave's azimuthal angle of propagation. The azimuthal angle can in general vary as the wave propagates, because plasma properties vary in directions perpendicular to the field lines, and components of the $\vec{n}$-vector in these directions are also allowed to vary. (This holds true in a tokamak geometry, but not necessarily so in a simple plane-stratified geometry.) The azimuthal angle is undetermined, and a specific value of $n_{\|}$ is unknown in the present analysis. But a range of $n_{\|}$values is now shown to be admissible: $n_{\|}$can now upshift or downshift. In the present case, however, the solution curve is finite 
in its spatial extent, and there is an upper bound to $n_{\|}$upshifting, and a lower bound to $n_{\|}$downshifting. All admissible solutions have a positive $n_{\|}$, and no waves travelling in the negative toroidal direction can be present; toroidal reflection of waves is not permitted. We note also that waves of all azimuthal angles of propagation $\left(-\pi<\chi_{n} \leq \pi\right)$ are admitted (see Fig. $14 \mathrm{c}$ ). But those waves with largest $n_{\|}$values propagate in, or near, the tangent plane in the positive $y$-direction $\left(\chi_{n} \sim+\pi / 2\right)$, i.e., in the positive poloidal direction (see Fig. 14a and b). We recall that the tilt of the solution curve in this direction is a consequence of the choice of a counter-parallel plasma current direction. At locations at which the G.C. plane zenith angle is greater than, but close to, the E.A. cone zenith angle, the ellipse is highly elongated, and waves with large $n_{\|}$values propagate in a narrow range of azimuthal angle along the local magnetic surface.

The solution surfaces are shown in Fig. 15 for the SUPRA model plasma. Both upper and lower halves of the $\vec{n}$-space are shown. The magnetic field-line pitch angle and oblique resonance angle are both large in this plasma. Consequently, the G.C. plane is tilted significantly off the horizontal, and the E.A. cone has a wide vertex angle. Under these circumstances, the oblique resonance zenith angle is outside the G.C. zenith angle $\left(\zeta_{\text {res }}>\zeta_{\text {geo }}\right)$, and the oblique resonance is now an admissible solution. The G.C. plane also intersects both upper and lower E.A. cones.

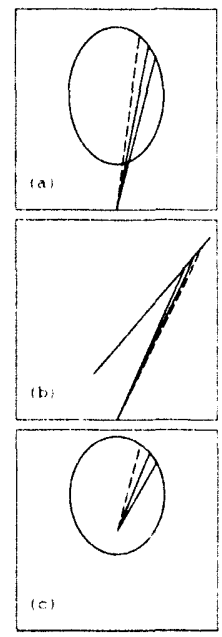

Fig. 14 The solution space curve and vectors of Fig. 13 are shown here in projections onto: (a) $n_{x}-n_{z}$ plane, (b) $n_{y}-n_{z}$ plane, and (c) $n_{x}-n_{y}$ plane.

The intersections of the G.C. plane and E.A. cones are two planar space curves, which are a hyperbola in their own plane. The space curves are presented in the same 3D $\vec{n}$-space in Fig. 16. A vector drawn from the origin to any point on either of the curves represents an admissible solution. A few such vectors are indicated by solid and broken lines in the figure. The origin of the vector space is at the center of the 'box' shown in the figure. Projections of the space curves onto (a) $n_{x}-n_{z}$ plane, (b) $n_{y}-n_{z}$ plane, and (c) $n_{x}-n_{y}$ plane are shown in Fig. 17.

The nature of wave propagation is qualitatively different between the conditions described in Fig. 12 and Fig. 15. The origin of the difference is whether or not the oblique resonance is an admissible solution, or the resonance is 'realizable' at the spatial point under consideration. Transitions between the two sets of conditions can take place within a plasma (e.g., the SUPRA model plasma). 
The hyperbolic solution curves are infinite in their spatial extent, and thus the range of admissible values of $n_{\|}$is also infinite. There is no upper bound to $n_{\|}$upshifting imposed by the G.C. relation. Admissible solutions now include both positive and negative $n_{\|}$, and waves travelling in either toroidal direction can be present. This suggests the possibility of toroidal reflection of waves. (The solution curves, representing positively and negatively travelling waves, do not touch each other, and wave reflection, if it indeed occurs, may therefore have to involve some non-adiabatic processes. Such processes may be provided, e.g., by density fluctuations. The subject is, however, beyond the scope of this article.) The reflected waves, travelling in the negative direction and possibly driving a current in the unwanted direction, can exist even in a perfectly uni-directional wave spectrum launched by an ideal grill, and are different from 'reverse waves' that are inevitably present in the spectrum of a real-life grill with an imperfect directivity ${ }^{5}$.

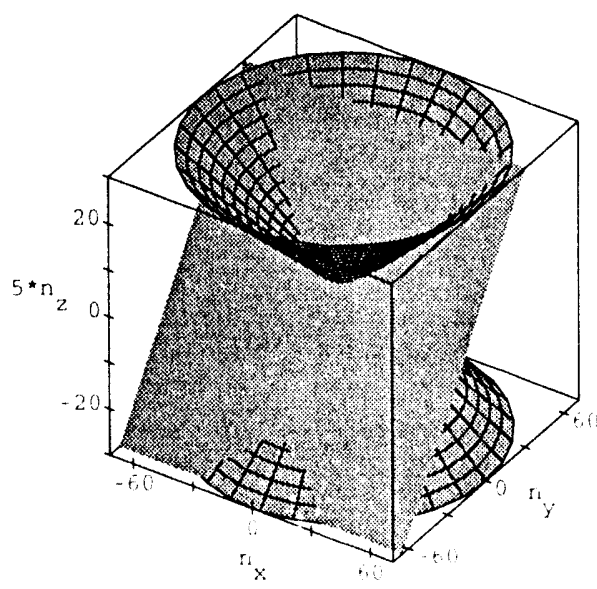

Fig. 15 The upper and lower E.A. cones, and G.C. plane are shown for a mid-radius point on the outboard midplane $\left(R_{m a j}=3.20 \mathrm{~m}\right.$ and $z=0 \mathrm{~m}$ ) of the SUPRA model plasma. A wave with a positive $n_{\|}$value $\left(n_{0}=21.8\right)$ is assumed to be launched from the plasma edge in the outboard midplane. The intersections of the (i.C. plane and E.A. cones are a hyperbola.

Waves characterized by hyperbolic solution curves propagate within two discrete ranges of azimuthal angle (see Fig. 1Ta): one for waves propagating in the positive toroidal direction and another for waves traveling in the negative toroidal direction. Within each range, waves with large $n_{\|}$values propagate in a 'beam-like' manner within two narrowly defined ranges of azimuthal angle (see Fig. ITa and b). Unlike waves described by an elliptic solution curve, these waves propagate at an angle to magnetic surfaces. Waves with small $n_{\|}$values may convert to the fast waves and may not be adequately described by the E.A. dispersion relation (see below).

Consider a wave at a point $\left(\vec{r}=\vec{r}_{1}\right)$ in space which has a specific refractive index vector $\left(\vec{n}=\vec{n}_{1}\right)$. When this wave propagates to a neighboring point $\left(\vec{r}_{2}\right)$, it assumes in general a different refractive index vector $\left(\vec{n}_{2}\right)$. The change in $\vec{n}$ is dictated by changes in the dispersion and G.C. relations, and by local gradients of the plasma parameters. For example, $\vec{n}$ may be

\footnotetext{
${ }^{5}$ We have unfortunately adopted the term, 'backward waves, in earlier publications ${ }^{17.26}$ to mean this unwanted part of a real grill spectrum. Wo will henceforth use the term, 'reverse waves.' for this purpose, and, following a more common usage, reserve the term, 'backward waves,' to mean waves having the phase and group velocities in an opposite direction.
} 
rotated through an azimuthal angle under the action of gradients as well as through a zenith angle under changing dispersion. If $\vec{n}_{2}$ is close to the oblique resonance at the new point, the change in the refractive index vector $\left(\delta \vec{n} \equiv \vec{n}_{1}-\vec{n}_{2}\right)$ can be large for an arbitrarily small change in position $\left(\delta \vec{r} \equiv \vec{r}_{1}-\vec{r}_{2}\right)$. (Reference to Figs. 16 and 17 is helpful in this discussion.) The fundamental requirement of the WKB approximation can thus be violated wherever the oblique resonance is realizable.

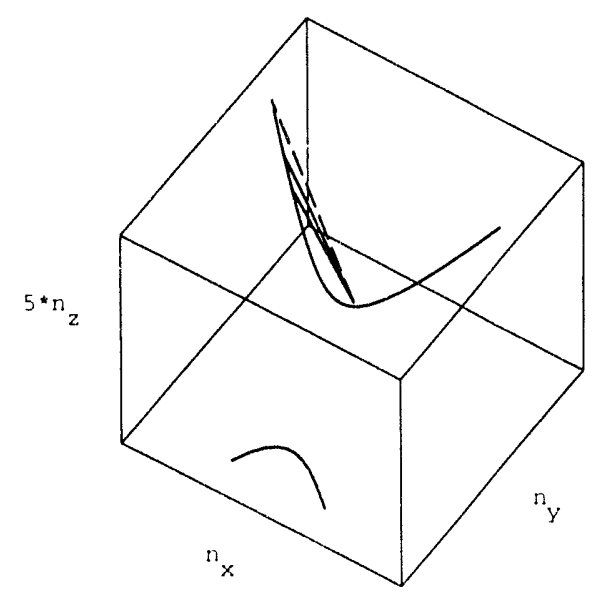

Fig. 16 The intersections of the E.A. cones and G.C. plane are shown for the same conditions as in Fig. 15. The intersections are two planar space curves, which are a hyperbola in their own plane. A few vectors drawn from the origin to points on the curves are indicated by solid and broken lines. The origin of the vector space is located at the center of the 'box.' These vectors represent admissible solutions.

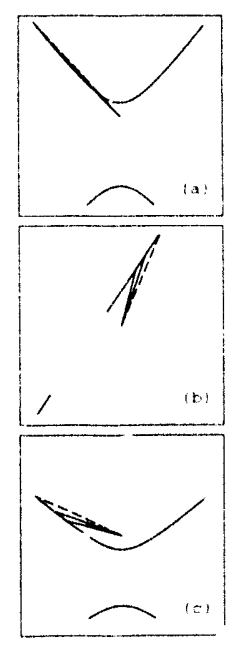

Fig. 17 The solution space curve and vectors of Fig. 16 are shown here in projections onto: (a) $n_{x}-n_{z}$ plane, (b) $n_{y}-n_{z}$ plane, and (c) $n_{x}-n_{y}$ plane.

Both the magnetic field-line pitch angle and oblique resonance angle are small in the TRIAM model plasma. (We recall that the lemniscoid wave normal surface in this model plasma belongs to the bounded volume, ' $T$,' and has an oblique resonance quite far from the perpendicular.) Conserinently, the G.C. plane is nearly horizontal, and the E.A. cone has a narrow vertex angle. These surfaces are qualitatively the same as those shown in Fig. 13. The admissible solution curves are nearly a circle. There are no preferred azimuthal directions, and waves travel nearly isotropically in the plane perpendicular to the local field line. The range of admissible $n_{\|}$ is very small, and the extent of n.| upshifting or downshifting is severly limited under these conditions. 
The full fourth degree dispersion relation and G.C. relation are represented in a $3 \mathrm{D} \vec{n}$ space in Fig. 18 in order to examine solutions for small values of $n_{\perp}$ (or $r_{\|}$). (See Fig. 9 for comparison of the full and approximate solutions.) A part of the upper half space near the origin is shown in an expanded scale. The salient feature in the middle of the otherwise cone-like surface represents the fast wave solution. Intersections of the full dispersion relation surface and G.C. plane can be quite complex in this region. A 2D vertical cross section of these surfaces is shown in Fig. 19. The cross section is along the tangent plane: the $\mathrm{x}$-axis of the local coordinate system, which is perpendicular to a magnetic flux surface and pointing 'radially' into the volume enclosed by the surface, comes out of the plane of the page. The positive axis denoted by $n_{p p}$ in the figure is the positive $n_{y}$-axis.

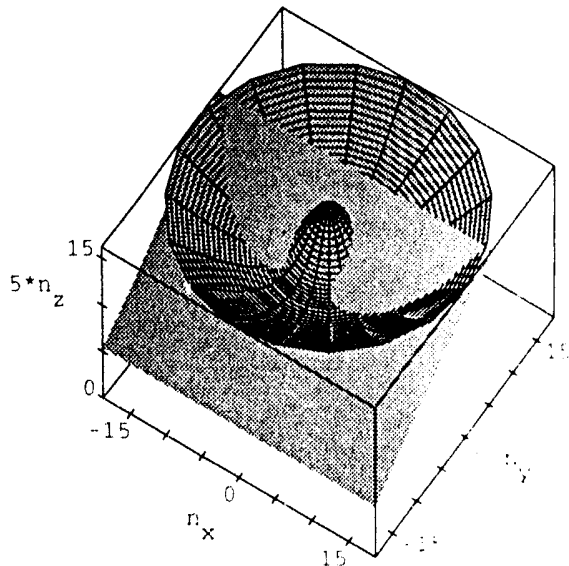

Fig. 18 The full dispersion relation surface and G.C. plane are shown for a mid-radius point on the inboard midplane $\left(R_{m a j}=1.50 \mathrm{~m}\right.$ and $Z=0)$ in the PBXM model plasma. Only the upper half space is shown. The salient feature in the middle of the otherwise cone-like surface represents the fast wave solution.

The two thick curves in lig. 19 represent the full dispersion relation. The bottom of the upper curve, or the top of lower curve (indicated, e.g., by ' $\mathrm{M}$ ' on the lower curve), is determined by the mode conversion condition. and the value of $n_{\|}$there is given by Eq. 5 . At the intercept of the dispersion relation curres and $n_{\|}$axis (indicated, e.g., by ' $C$ ' on the lower curve), the value of $n_{\|}$satisfies the last wave cutoff condition given by Eq. 7 . Broken lines through the origin represent the E.A. cone's. 'The solid line through the point, 'P,' represents the G.C. plane. The intercept point. 'P.' roflects the 'wedge effect,' and $n_{\|}$there is given by Eq. 14. A space curve passing through points. ' $a$ ' and ' $b$,' represents the simultaneous solutions to the full dispersion relation and (i.C'. relation. When the $n_{\|}$-axis intercept, 'P,' of the G.C. plane, is smaller in magnitude than $n_{\|}$at the fast wave cutoff $\left(n_{\|}{ }^{u d g}<n_{\|}{ }^{F C}\right)$, there are propagating fast waves. The solution curve is now an ellipse-like curve truncated at the bottom. The points, 'a' and 'b,' in Fig. 19 define the range of admissible $n_{\|}$. In particular, the value of $n_{\|}$ at the point, 'a,' is the upper bound of the $n_{\|}$upshifting. It is thus this tilting of the G.C. plane that can produce a far more significant $n_{\|}$upshifting than the 'wedge effect.'

We use Fig. 19 to describe transitions between the two sets of conditions shown in Fig. 12 and Fig. 15. If the tilt of the G.C. plane from the horizontal becomes stronger $\left(\zeta_{\text {geo }}\right.$ becomes smaller), the point, 'a,' would move upward along the upper dispersion relation curve, and the range of admissible $n_{\|}$would become larger. Finally, it would disappear to $+\infty$, when $\zeta_{g e o}$ 
exactly equals $\zeta_{\text {res }}$. The point, 'a,' would reappear at $-\infty$, and move upward along the lower dispersion curve into finite $n_{\|}$regions, as $\zeta_{\text {geo }}$ becomes smaller than $\zeta_{\text {res }}$. (Waves may become damped, as $n_{\|}$becomes large. Thus, toroidal reflection of waves, if it indeed occurs, must take place in a time scale short compared with Landau damping time scale.)

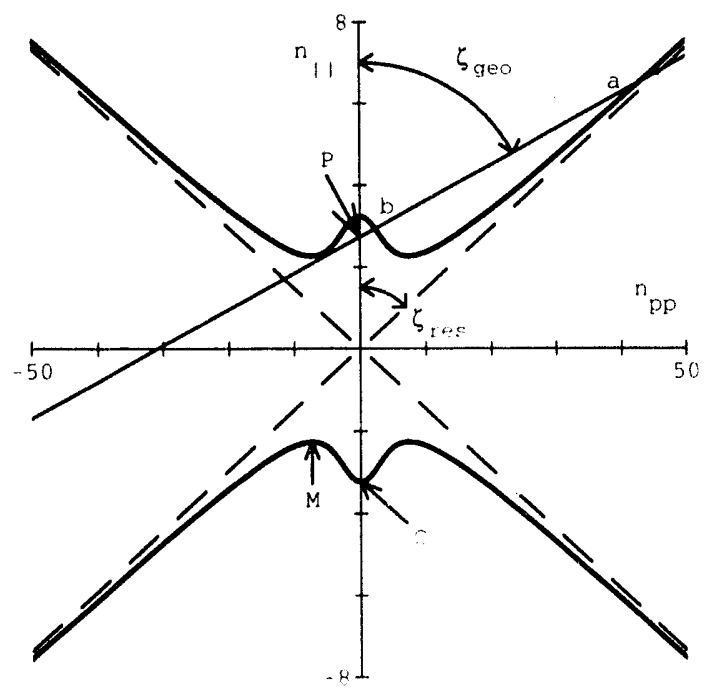

Fig. 19 A 2D vertical cross section along the tangent plane of the 3D surfaces in the previous figure is shown. The $n_{x}$-axis comes out of the plane of the page, and the positive $n_{p p}$ axis is the positive $n_{y}$-axis. The two thick curves represent the full dispersion relation. Broken lines through the origin represent the E.A. cones. The solid line, through an $n_{\|^{-}}$ intercept point, 'P,' represents the G.C. plane. A space curve passing through points, 'a' and 'b,' is the simultaneous solution to the full dispersion relation and G.C. relation.

We summarize this subsection. When the component of the $\vec{n}$-vector that is perpendicular to the magnetic field line is purcly in the direction of density inhomogeneity (i.e., perpendicular to the magnetic surface). lhis vector component has no projection onto the toroidal direction. When this vector componcint is rotated about the field line through an azimuthal angle, it casts a finite projection on to the toroidal direction. In order to maintain the toroidal mode number invariant, the component of the $\vec{n}$-vector that is parallel to the magnetic field line must also cast a compensating amount of projection onto the toroidal direction. When the perpendicular component casts a large projection onto the toroidal direction, the parallel component also casts a large compensating projection. This is the central feature of the $n_{\|}$upshifting mechanism described by Schuss, et al. ${ }^{10}$ In the present study, it was shown furthermore that, for large enough projections, the zenith angle of wave propagation can now match that of the oblifue resonance when the wave propagate at some azimuthal angles. The oblique resonance then becomes realizable, and the nature of wave propagation becomes qualitatively different. In a later section, we will describe another mechanism through which the perpendicular componcnt of the $\vec{n}$-vector and density gradient vector become 'misaligned' with each other, and 1 upshifting can occur.

\section{B. Bounds of $r_{\|}$Shifting}

A simultaneous solution to the E.A. dispersion relation, Eq. 11, and G.C. relation, Eq. 14, can be obtained analytically. Solving these equations for $n_{\|}$as a function of the wave propagation azimuthal angle, $\backslash_{n}$, we obtain,

$$
n_{\|}=\left(\frac{n_{\phi}}{\cos \zeta_{t}}\right) \frac{1}{1 \pm g \sin \chi_{n}}
$$


where the plus sign is for the co-parallel plasma current, and the minus sign is for the counterparallel plasma current. The 'g-factor' in this equation defined by,

$$
\begin{aligned}
g & \equiv \frac{\tan \zeta_{\text {res }}}{\tan \zeta_{\text {geo }}}, \\
& \left.=\left(\tan \zeta_{t}\right) \sqrt{(}-P / S\right)
\end{aligned}
$$

is the tangent ratio of the oblique resonance zenith angle to the tilt zenith angle of the G.C. plane, and is a positive quantity. Equation 17 gives the value of $n_{\|}$anywhere in the plasma under the electrostatic approximation, but only if the azimuthal angle, $\chi_{n}$, is specified.

The sufficient condition that $n_{\|}$be bounded for all azimuthal angles is given by,

$$
g<1
$$

When $n_{\|}$is bounded, the extreme $n_{\|}$shifting occurs in the tangent plane. Note that there is a preferred poloidal direction for the extreme $n_{\|}$upshift or downshift, and that the direction depends on the relative sense of the toroidal field and plasma current. Such preferences have been demonstrated in ray tracing calculations by Bernabei, et al., ${ }^{27}$ and provided foundation for experimenting different poloidal locations of the antenna. For a co-parallel plasma current, the extreme upshift occurs in the positive $y$-direction $\left(\chi_{n}=+\pi / 2\right)$, and the extreme downshift occurs in the negative $y$-direction $\left(\chi_{n}=-\pi / 2\right)$. For a counter-parallel plasma current, the extreme upshift occurs in the negative $y$-direction $\left(\chi_{n}=-\pi / 2\right)$, and the extreme downshift occurs in the positive $y$-direction $\left(\chi_{n}=+\pi / 2\right)$. The values of the extreme $n_{\|}$are given by,

$$
n_{\|}^{u p, d n}=\left(\frac{n_{\phi}}{\cos \zeta_{t}}\right) \frac{1}{1 \pm g} .
$$

The minus sign in the denominator corresponds to the extreme upshift $\left(n_{\|}^{u p}\right)$, and the plus sign to the extreme downshift $\left(n_{\|}^{d n}\right)$ for either a co- or counter-parallel plasma current.

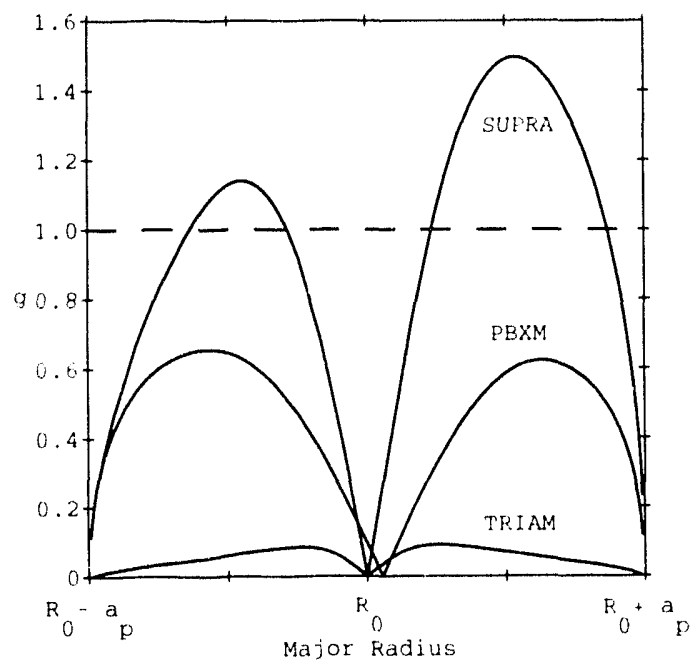

Fig. 20 'The 'g-factor,' which is the tangent ratio of the zenith angle of the oblique resonance to the zenith angle of the G.C. plane tilt, is plotted along the midplane. For both PBXM and TRIAM model plasmas, $g<1$, and $n_{\|}$upshift is bounded everywhere. For the SUPRA model plasma $g>1$ over a major part of the plasma volume, and $n_{\|}$upshift can be unbounded.

The solution for $n_{\|}$becomes infinite when the denominator of Eq. 17 vanishes, which can happen only if $g \geq 1$. The G.C. relation thus does not limit $n_{\|}$, when $g \geq 1$. But this is a 
necessary condition for unbounded $n_{\|}$. There may be other mechanisms that limit $n_{\|}$. The solution becomes infinite at azimuthal angles of propagation specific to the spatial point under consideration. The angles are given by,

$$
\sin \chi_{n}^{\infty}= \pm \frac{1}{g}
$$

where the plus sign is for a counter-parallel plasma current, and the minus sign is for a coparallel plasma current. (In ray tracing calculations, a 'truly unbounded ray' will be obtained only if the angle $\chi_{n}^{\infty}$ happens to be realized. Solutions for $n_{\|}$in the vicinity of the angle will, however, be very large.)

Variation along the midplane of the $g$-factor defined in Eq. 18 is shown in Fig. 20 for the three model plasmas. For both PBXM and TRIAM model plasmas, $n_{\|}$is bounded everywhere inside the plasma (the maximum $g$ value occurs in the midplane for a simple density profile). For the SUPRA model plasma, $n_{\|}$upshifting can be unbounded over most of the plasma volume. Using the approximate expressions for the dielectric tensor elements, Eq. 2, we write $-P / S \approx\left(\omega_{p e} / \omega\right)^{2} /\left(1+\left(\omega_{p e} / \omega_{c e}\right)^{2}\right)$. We note that $\tan \zeta_{t}=B_{\theta} / B_{\phi}$, and that usually $\left|\cos \zeta_{t}\right| \approx 1$. Using also Eq. 12 for the 'wedge effect', we write the upper bound of $n_{\|}$upshifting, normalized by the toroidal refractive index at the grill, as

$$
\begin{aligned}
\frac{n_{\|^{u p}}^{u p}}{n_{\phi}^{g r}} & \approx\left(\frac{R_{m a j}^{g r}}{R_{m a j}}\right) \frac{1}{1-g}, \\
g^{2} & \approx\left(\frac{B_{\theta}}{B_{\phi}}\right)^{2} \frac{\left(\omega_{p e} / \omega\right)^{2}}{1+\left(\omega_{p e} / \omega_{c e}\right)^{2}} .
\end{aligned}
$$

This is the approximate result reported earlier. ${ }^{17}$

The simple analytic form for the g-factor in Eq. 22 clearly shows under what conditions strong $n_{\|}$upshifting can be expected:

- a large magnetic field-line pitch angle, $B_{\theta} / B_{\phi}$,

- a large normalized density, $\left(\omega_{p e} / \omega\right)^{2}$, and

- to a lesser extent, a strong 'electron magnetization,' or small $\left(\omega_{p e} / \omega_{c e}\right)^{2}$.

We used earlier the term, 'dimensionless density,' to mean combined effects of the second and third items in the form, $\left(\omega_{p e} / \omega\right)^{2} /\left(1+\left(\omega_{p e} / \omega_{c e}\right)^{2}\right)$. Some results of ray tracing calculations are immediately understandable in terms of these expectations. For example, in comparison with the PBXM model plasma, the SUPRA model plasma shows ${ }^{16}$ stronger $n_{\|}$upshifting. due to its smaller aspect ratio that leads to larger magnetic field-line pitch angles, and its larger normalized density. The PBXM model plasma also has weak upshifting, because its low toroidal magnetic field results in a weak electron magnetization. The PBXM model plasma, which is bean-shaped, shows stronger upshifting than a circular plasma in the same tokamak, because the shaping results in larger field-line pitch angles. 


\section{Wave Domain and Unbridgeable Spectral Gap}

In previous and this sections, several formuli were developed for delimiting a range of $n_{\|}$ in which some specific characteristics hold. They are the mode conversion (Eq. 5), fast wave cutoff (Eq. 7), lower limit of $n_{\|}$downshifting, and the upper limit of $n_{\|}$upshifting (Eq. 20). Variations of these bounds along the midplane are plotted in Fig. 21 for the PBXM model plasma. The 'wedge effect' is also indicated (Eq. 12). The wave launched by the grill is $n_{\phi}^{g r}=2.1$. These bounds are simultaneously applicable, and thus the lowest upper bound and highest lower bound define an admissible range of $n_{\|}$at each point in space. $A$ variation of the admissible range of $n_{\|}$as a function of position defines a 'wave domain.'

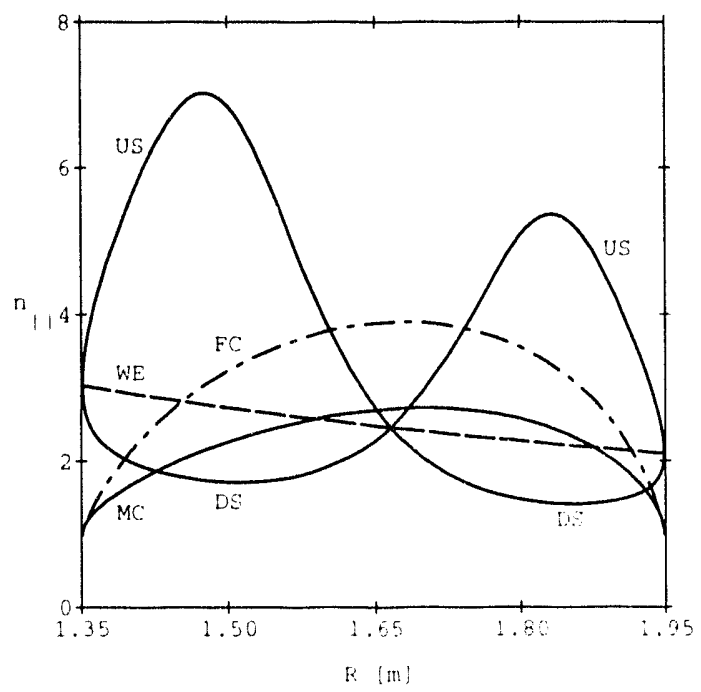

Fig. 21: Various bounds on $n_{\|}$is plotted along the midplane for the PBXM model plasma: the upper bound of $n_{\|}$upshifting (US), lower bound of $n_{\|}$downshifting (DS), mode conversion (MC), and fast wave cutoff (FC) are shown. The 'wedge effect' (WE) is also indicated. The wave launched by the grill is $n_{\phi}^{g r}=2.1$.

Waves with a high $n_{\|}$value will damp strongly through electron Landau damping. The condition that the wave phase speed be a certain multiple, $\lambda$, of the electron thermal speed can be expressed as,

$$
n_{\|}^{d m p}=\frac{c}{\lambda v_{\epsilon}}
$$

For the phase speed equal to three times the thermal speed $(\lambda=3)$, the damping is strong. The damping is exponentially weaker at a higher phase speed. Six times the thermal speed $(\lambda=6)$ may somewhat arbitrarily be taken as a linit of minimal but finite damping.

The wave domain for a plasma with bounded $n_{\|}$upshifting is a closed volume in a $3 \mathrm{D}$ space defined by $n_{\|}$and the spatial coordinates in the plasma cross section. A constant Landau damping (LD) condition can be represented by a surface in the same 3D space. A cross section of the wave domain and three Landau damping surfaces through the midplane is shown in Fig. 22 for the PBXM model plasma with the grill-defined $n_{\phi}^{g r}=2.1$. The lower hybrid waves exist within the entire wave domain, but the fast waves exist only regions below the fast wave cutoff. The wave domain is larger on the inboard side than on the outboard side, but effects of wave damping on electrons are expected to be homogenized on each magnetic flux surface. The broken lines labeled $\lambda=3, \lambda=4$ and $\lambda=6$ represent Landau damping surfaces, Eq. 23, for strong, weak and minimal damping, respectively. Overlapping of the wave 
domain and minimal damping surface (region above $\lambda=6$ curve) indicates that the waves are marginally damped. The spectral gap, between the wave spectrum and thermal part of the electron parallel velocity distribution, can barely be bridged in this plasma, even if the maximum possible $n_{\|}$upshifting indeed takes place.

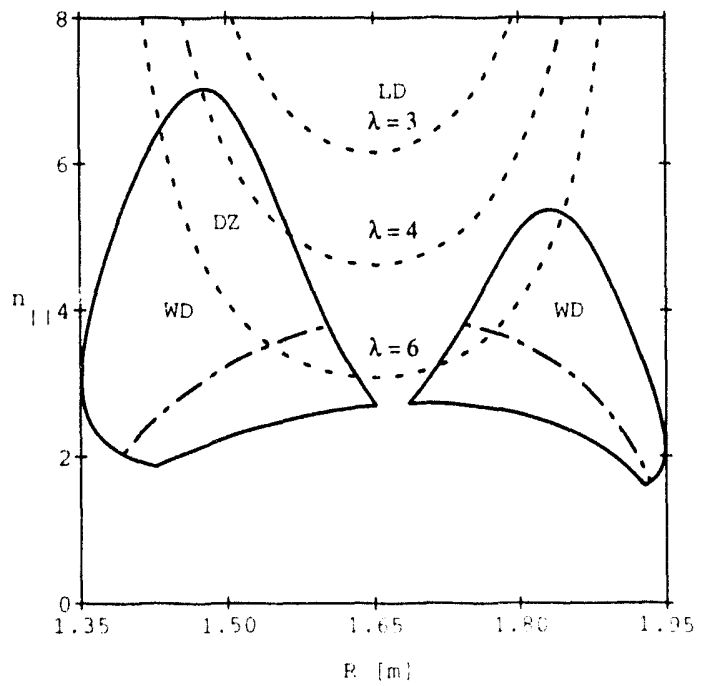

Fig. 22: The wave domain (WD) and Landau damping surfaces (LD) are shown in a 2D cross section for the PBXM model plasma. The fast wave cutoff is also shown by a broken line. A space common to the wave domain and interior of a damping curve is the damping zone (DZ).

Variations of the upper bound of $n_{\|}$upshifting, 'wedge effect,' and four Landau damping surfaces $(\lambda=3,4,6$ and 10$)$ are shown in Fig. 23 for the TRIAM model plasma with the grilldefined $n_{\phi}^{g r}=1.8$. The maximum possible $n_{\|}$upshifting is extremely weak for this plasma, because of small pitch angles and low densities. The maximum possible $n_{\|}$is not large enough by a big margin to cause the waves to damp anywhere within the plasma. For a Landau damping surface to intersect the wave domain, $\lambda=14$ is required. There is thus a huge spectral gap that cannot be bridged for this model plasma. This conclusion, which is a direct and inevitable consequence of the assumed invariance of the toroidal mode number, is, however, in contradiction to an experimental fact: the current in the TRIAM-1M tokamak was driven entirely by LHCD in the experiment emulated by the model plasma.

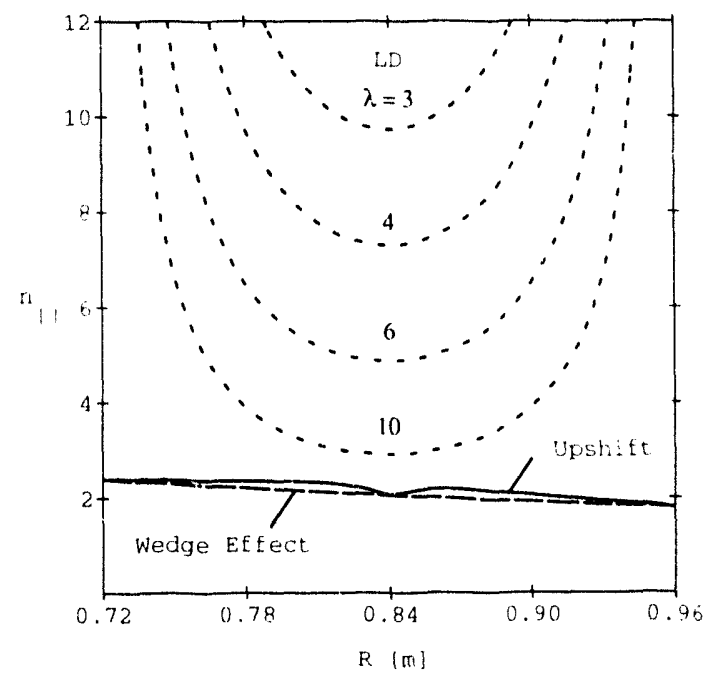

Fig. 23: Variations of the upper bound of $n_{\|}$ upshifting, wedge effect, and Landau damping surfaces (LD) are shown for the TRIAM model plasma. For a Landau damping surface to intersect the wave domain for this plasma, $\lambda=14$ is required. 
For the SUPRA model plasma, the requirement of the toroidal mode number invariance does not impose an upper bound to $n_{\|}$upshift over most of the plasma volume. But ray tracing calculations by Kupfer and Moreau ${ }^{16}$ showed the presence of an upper bound to a chaotic ray distribution along a magnetic flux surface at some mid-range radius (not specified). The bound cannot, however, be due to the invariance requirement (assuming that the chosen flux surface belonged to a region with $g \geq 1$ ).

\section{D. $n_{\|}$Upshifting caused by Parallel Density Gradient}

We have shown in previous subsections that $n_{\|}$upshifting produced by the finite pitch angle of magnetic field lines can be bounded and insufficient to explain experimentally observed wave damping. The geometrical interpretation in terms of 'tilting' of the C.C. plane introduced in those subsections suggests that there may be other physical mechanisms that lead to such tilting. We introduce in this subsection an $n_{\|}$upshifting mechanism that is caused by the density gradient along magnetic field lines. Such a gradient may exist locally in the plasma equilibrium state under special circumstances, or may arise temporarily and locally due to MHD perturbations or density fluctuations.

In linear experimental devices, the plasma density varies predominantly in the radial direction perpendicular to magnetic field lines. But it is often the case that there are also small density variations along the field lines. Effects of a parallel density gradient on lower hybrid wave propagation were examined theoretically by Briggs and Parker ${ }^{18}$ in connection with an experimental study of resonance cones. They considered a plane-stratified condition in which the density gradient was uniformly 'tilted' from a direction perpendicular to uniform field lines $\left(\vec{B} \cdot \nabla n_{e} \neq 0\right)$, and found that waves were absorbed at a 'new' absorption layer some 'distance' (in terms of the density) away from the lower hybrid resonance layer. The distance was related to the magnitude of the density gradient tilt. These effects were also investigated experimentally by Bellan and Porkolab ${ }^{28}$ in a study of resonance cones. The 'image' of a periodic antenna structure was carried along the characteristics that ran essentially parallel to each other in the presence of only a weak parallel density gradient. The characteristics became 'bunched' as the parallel density gradient was increased-the periodicity of the image became progressively smaller as the wave propagated axially into a region of lower densities. We will show that the 'new' absorption layer in the analysis of Briggs and Parker is a manifestation of the oblique resonance, and furthermore that a density gradient along field lines can lead to $n_{\|}$upshifting in a more complex tokamak geometry.

We consider a plane-stratified plasma in the next several paragraphs: the magnetic field is uniform, and the density rises monotonically from zero at the plasma edge to values high enough to satisfy the lower hybrid resonance condition in an interior point. By 'inverse Fourier transforming' Eq. 11, we write the differential equation governing the wave potential, $\varphi$, of lower hybrid waves as,

$$
\nabla_{\perp}\left(S \nabla_{\perp} \varphi\right)+\frac{\partial}{\partial z}\left(P \frac{\partial}{\partial z} \varphi\right)=0
$$

where $\nabla_{\perp} \equiv \hat{x} \partial / \partial x+\hat{y} \partial / \partial y$. The magnetic field is in the $z$-direction. The density gradient 
has components both in the $x$ - and $z$-directions: the gradient vector is in the $z$ - $x$ plane, and is tilted by an angle, $\alpha$, measured from the $x$-direction. Positive values of $\alpha$ is in the direction going from the positive $z$-axis toward the positive $x$-axis.

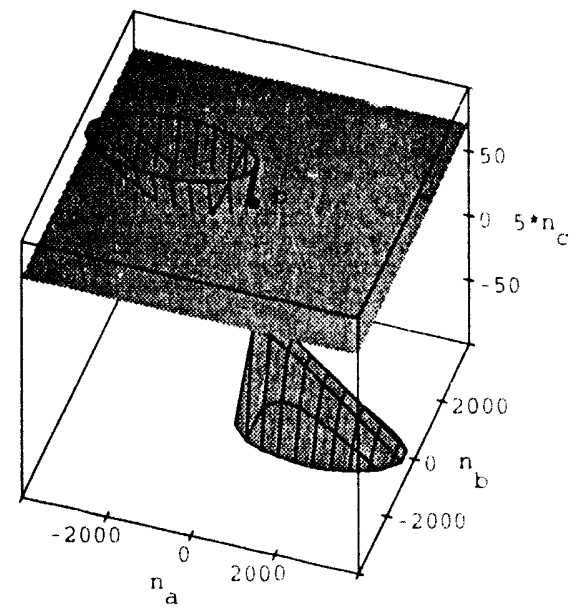

Fig. 24 Surfaces of the E.A. dispersion relation and G.C. relation are shown in the $\left(n_{a}, n_{b}, n_{c}\right)$-space for a 'low density' point, A, at which the density gradient is 'tilted' from a direction perpendicular to the magnetic field. The dispersion relation is represented by a pair of tilted cones, and the G.C. relation is a horizontal plane. The intersection of these surfaces is an ellipse with its center displaced in the negative $n_{a}$-axis direction.

We define a right-handed rectangular coordinate system, $(\hat{a}, \hat{b}, \hat{c})$, that is rotated from the original coordinate system, $(\hat{x}, \hat{y}, \hat{z})$, about the $y$-axis by $\alpha$. The $y$ - and $b$-axes are aligned with each other. We also designate by $\xi$ the azimuthal angle about the $c$-axis. It ranges over $-\pi<\xi \leq \pi$, and its positive values are measured from the positive $a$-axis toward the positive $b$-axis. Transforming the derivatives through $\partial / \partial x=\cos \alpha \partial / \partial a+\sin \alpha \partial / \partial c, \partial / \partial y=\partial / \partial b$, and $\partial / \partial z=\cos \alpha \partial / \partial c-\sin \alpha \partial / \partial a$, the governing equation in the new coordinate system is,

$$
\frac{\partial}{\partial a}\left(\epsilon_{a a} \frac{\partial \varphi}{\partial a}\right)+\epsilon_{b b} \frac{\partial^{2} \varphi}{\partial b^{2}}+\epsilon_{c c} \frac{\partial^{2} \varphi}{\partial c^{2}}+\frac{\partial}{\partial a}\left(\epsilon_{a c} \frac{\partial \varphi}{\partial c}\right)+\epsilon_{a c} \frac{\partial^{2} \varphi}{\partial a \partial c}=0
$$

where

$$
\begin{aligned}
\epsilon_{a a} & \equiv S \cos ^{2} \alpha+P \sin ^{2} \alpha, \\
\epsilon_{b b} & \equiv S, \\
\epsilon_{c c} & \equiv P \cos ^{2} \alpha+S \sin ^{2} \alpha, \\
\epsilon_{a c} & \equiv(S-P) \sin \alpha \cos \alpha .
\end{aligned}
$$

Components of the refractive index vector in the two coordinate systems are related through a transformation: $n_{a}=n_{x} \cos \alpha-n_{z} \sin \alpha, n_{b}=n_{y}$, and $n_{c}=n_{z} \cos \alpha+n_{x} \sin \alpha$. Because no plasma properties vary in the $b$ - and $c$-directions, we may use Fourier analysis in these directions, and write $\varphi(a, b, c)=\varphi_{0}(a) \exp -i\left(n_{b}+n_{c}\right)$. We obtain an ordinary differential equation,

$$
\frac{d}{d a}\left(\epsilon_{a a} \frac{d \varphi_{0}}{d a}\right)-\epsilon_{b b} n_{b}^{2} \varphi_{0}-\epsilon_{c c} n_{c}^{2} \varphi_{0}-i n_{c}\left(\frac{d}{d a}\left(\epsilon_{a c} \varphi_{0}\right)+\epsilon_{a c} \frac{d \varphi_{0}}{d a}\right)=0 .
$$


This is an equation identical ${ }^{6}$ to the one obtained by Briggs and Parker, ${ }^{18}$ except that we extend the analysis to a $3 \mathrm{D} \vec{n}$-space, and consider a range of $n_{b}$. (They considered a 2D space, and hence a specific value of $n_{b}=0$, or $\xi=0$ or $\pi$.) They have shown, in a manner analogous to Stix's analysis of the lower hybrid resonance, ${ }^{23}$ that wave absorption through mode conversion occurs where the coefficient of the highest order derivative term vanishes, i.e., $\epsilon_{a a}=0$, or $\pi / 2-\alpha=\arctan \sqrt{(}-P / S)$. But this is identical to the condition under which the oblique resonance occurs, Eq. 9: when the zenith angle, $\pi / 2-\alpha$, that the density gradient makes with respect to the $z$-axis equals the oblique resonance zenith angle, $\left.\zeta_{\text {res }}=\arctan \sqrt{(}-P / S\right)$, wave absorption occurs.

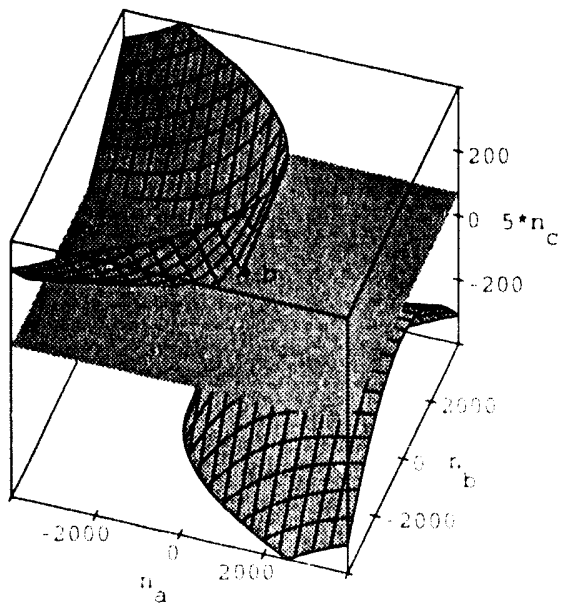

Fig. 25 Surfaces of the E.A. dispersion relation and G.C. relation are shown for a 'medium density' point, B. The dispersion relation is represented bv a pair of tilted cones with a vertex angle wider than that for cones shown in Fig. 24. The intersection of these surfaces is a part of a hyperbola in the negative $n_{a}$ halfspace. The condition shown here correspond to the 'new' absorption layer of Briggs and Parker.

When effects of the inhomogeneity on the dielectric tensor elements themselves, i.e., $d \epsilon_{a a} / d a$ and $d \epsilon_{a c} / d a$ terms, are neglected (i.e., a homogeneous dispersion relation is used, but effects of the tilted density gradient is included), the E.A. dispersion relation is given by,

$$
\epsilon_{a a} n_{a}^{2}+\epsilon_{b b} n_{b}^{2}+\epsilon_{c c} n_{c}^{2}+2 \epsilon_{a c} n_{a} n_{c}=0
$$

Because no plasma properties vary in the $c$-direction, we may assume that the refractive index component, $n_{i}$, remains constant as waves propagate. The geometrical constraint relation is then given by,

$$
n_{c} \equiv n_{z} \cos \alpha-n_{x} \sin \alpha=n_{c}^{0},
$$

where $n_{c}^{0}$ is a constant.

The dispersion relation and G.C. relation are plotted in Figs. 24 through 26 for three spatial locations, $\mathrm{A}, \mathrm{B}$, and $\mathrm{C}$, that are progressively closer to the lower hybrid resonance layer. The E.A. dispersion relation in the new coordinate system is a tilted conc (as seen most evidently in Fig. 24) and the geometrical constraint relation is a horizontal plane in these figures. As in the analysis in the previous sections, the intersection of these surfaces represents admissible

\footnotetext{
${ }^{6}$ Equation (5) of the cited reference is written as a partial differential equation. From the context, however, an ordinary differential equation is appropriate.
} 
solutions. At the point, A, the tilt zenith angle is greater than the cone vertex angle, i.e., $\pi / 2-\alpha>\zeta_{\text {res }}$, and the intersection of these surfaces is an ellipse with its center displaced in the negative $a$-axis direction. Finite solutions exist for the entire range of $\xi$ at this point: all components of the $\vec{n}$-vector, including in particular $n_{\|}$, are therefore bounded for all values of $\xi$. Points at which the solution curve intersects the $n_{b}=0$ plane, indicated by ' $b$ 's' in the figure, are the solutions obtained by Briggs and Parker ${ }^{18}$ in their $2 \mathrm{D}$ analysis. They termed these solutions two 'modes,' although they are in fact the 'same' wave propagating at two different azimuthal angles. The qualitative behavior of these points as the density varies was sketched in Fig. 3 of the cited reference. The vertex angle of the tilted cones becomes wider at interior points. One of the ' $b$ points' moves toward large negative $n_{a}$ values, and then disappears to $n_{a}=-\infty$. At the point, $\mathrm{B}, \pi / 2-a=\zeta_{\text {res }}$, and the intersection consists of that part of a hyperbola in the negative $n_{a}$ half-space (Fig. 25). Admissible solutions are found only over a limited range of $\xi$ at this point. The 'new' absorption laver of Briggs and Parker corresponds to this point. They showed that one of the 'modes' propagates through the layer, and the other is absorbed at the layer. At the point, $\left(', \pi / 2-a<\zeta_{\text {res }}\right.$, and the intersection is a hyperbola (Fig. 26). Admissible solutions are found over two disconnected ranges of $\xi$ at this point. At all locations between the point, $C$. and the lower hybrid resonance layer, the solutions are qualitatively the same as those at $\mathrm{C}$. but the two halves of the hyperbola are closer together. At the hybrid resonance (not shown), the two 'b points' will coalesce at the point, $n_{a}=n_{c}^{0} / \tan \alpha, n_{b}=0$. and $n_{c}=n_{c}^{0}$. Briggs and Parker stated that waves are reflected at the lower hybrid layer (in the presence of a tilted density gradient).

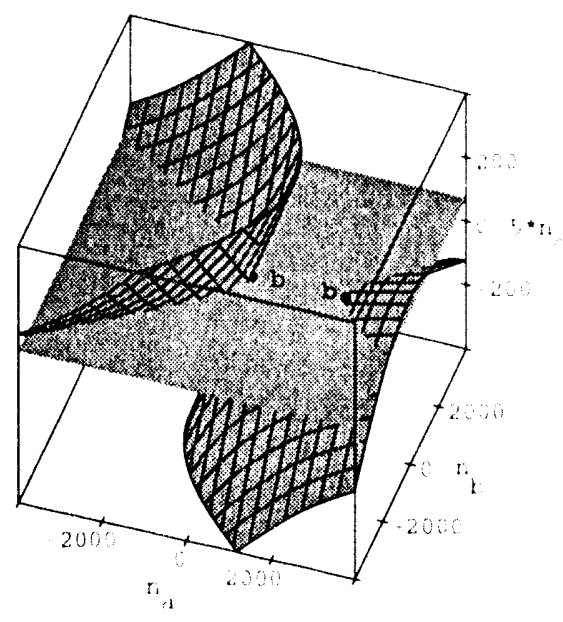

Fig. 26 Surfaces of the E.A. dispersion relation and (i.c. relation are shown for a high density' point, ( $'$ 'The dispersion relation is represented by a pair of tilted cones with a vertex angle wider than that for cones shown in Fig. 24 or 25. The intersection of these surfaces is a hyperbola.

The above analysis in a plane-stratified geometry is not globally applicable to tokamaks, but may be approximately applicable locally to the tokanak geometr. We assume that the geometrical constraint condition is that the component of the $\vec{n}$-vector in a direction perpendicular to the local density gradient remains invariant. The foregoing analysis has been made in the $\left(n_{a}, n_{b}, n_{c}\right)$-space in order to show its relationship to the results of Briggs and Parker. ${ }^{18}$ But transformation back to the $\left(n_{x}, n_{y}, n_{z}\right)$-space makes the connection to the results obtained in earlier sections clear: the dispersion relation is still a pair of straight cones as in the previous sections, but the (i.C. relation is now a plane tilted in the $x-z$ (rather than $y$-z) plane. The new 
G.C. relation causes $n_{\|}$upshifting in a manner analogous to the G.C. relation that described the toroidal mode number invariance. The tilt angle can also become sufficiently large to make upshifting unbounded for some azimuthal angles of propagation. In the tokamak geometry, these unbounded 'admissible' solutions can actually be 'realized,' because the $\vec{n}$-vector rotates about the field line and can attain these azimuthal angles as waves propagate. (In the planestratified geometry considered above, unbounded solutions can occur only at a single point in space, because $n_{b}$ and $n_{c}$ are given, and the $\vec{n}$-vector does not rotate.)

The condition for the unbounded $n_{\|}$can be written as,

$$
\left.\left|\frac{\partial n_{e}}{\partial z}\right| /\left|\nabla_{\perp} n_{e}\right| \geq \sqrt{(}-\frac{S}{P}\right)
$$

Here, we consider again the right-handed local coordinate system. $(\hat{x}, \hat{y}, \hat{z})$, described in Sec. IV: the $x$-axis is perpendicular to the local magnetic surface pointing into the volume enclosed by the surface, the $z$-axis is in the surface, aligned with the local magnetic field, and the $y$-axis is also in the surface, pointing nearly in the poloidal direction. The equilibrium density gradient is in the positive $x$-direction. The clensity gradient tilt angle is $\alpha \equiv \arctan \left(\left(\partial n_{f} / \partial z\right) /\left|\nabla_{\perp} n_{\epsilon}\right|\right)$,

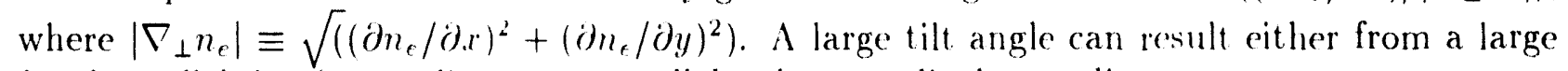
local parallel density gradiont. or a small local perpendicular gradient.

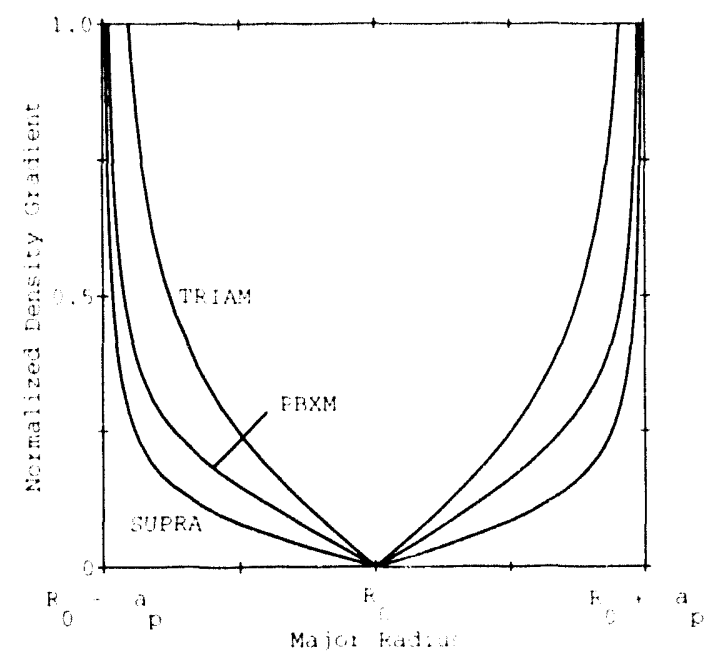

Fig. 27 The minimum parallel density gradient along magnetic fick lines needed to satisfy the unbounded $n_{\|}$upshifting condition. The parallel gradient is normalized by the average radial gradient, $n_{f}(0) / a_{p}$. A large parallel gradient may occur in the equilibrium profile under special circumstances. The needed gradient will be much smaller, if a local 'flat spot' is generated in the perpendicular profiles, e.g., by a density perturbation.

When the perpendicular density gradient in the denominator of Eq. 30 is the radial gradient of a parabolic equilibrium density profile, the parallel density gradient needed for unbounded $n_{\|}$upshifting. normalized by the average radial density gradient is,

$$
\left.\frac{a_{p}}{n_{e}(0)}\left|\frac{\partial n_{f}}{\partial z}\right| \geq \frac{2 r_{\min }}{a_{p}} \sqrt{(}-\frac{S}{P}\right)
$$

The minimum value of $\sqrt{(}-5 / P)$ is 7,14 , and $21 \%$ for the STPRA, PBXM and TRIAM model plasmas, respectively (see Table 2). Variations along the midplane of the RHS of this inequality is shown in Fig. 27. A parallel density gradient, that is a significant fraction of the 
average radial gradient, is needed to satisfy the unbounded $n_{\|}$upshifting condition. Although little experimental evidence exists, it is generally believed that rapid transport of heat and particles makes it difficult to maintain a large density gradient along field lines. Large parallel gradients may exist, however, under some special circumstances (see discussion in Sec. VI).

Density perturbations can alter the local density gradient. In a linear theory of drift waves or ballooning mode, the parallel wave number of the perturbation is zero, and there is no parallel density gradient. In electrostatic turbulence, mode coupling broadens the parallel wave number spectrum, ${ }^{29}$ and the parallel density gradient becomes finite. The gradient is theoretically expected to be small, $\left|\partial n_{e} / \partial x\right| \sim\left|\partial n_{e} / \partial y\right| \gg\left|\partial n_{e} / \partial z\right|$, although there have been no measurements of the parallel gradient. A significant tilt of the density gradient vector is then generated, only if the perpendicular gradient is small. When the perturbation amplitude is large enough, $\dot{n} / n_{e} \sim \lambda_{x} / \lambda_{n}$, where $\lambda_{n} \equiv n_{e} /\left(d n_{e} / d x\right)$ is the 'radial' gradient scale length, and $\lambda_{x}$ is the radial wave length of the perturbation, the local radial profile can become (nearly) flat. There are in general specific locations at which the 'poloidal' profile is also (nearly) flat, but at which the parallel gradient remains finite. At such points, the tilt angle becomes large, and upshifting can become unlimited. Density fluctuations that exist commonly in tokamaks and other devices can thus provide an $n_{\|}$upshifting mechanism.

\section{SUMMARY AND DISCUSSION}

Characteristics of lower hybrid wave propagation were studied in connection with the lower hybrid current drive (LH('D) in tokamaks through an analysis of a cold plasma dispersion relation and various geometrical constraint (C.('.) relations.

The dispersion relation has solutions for lower hybrid waves with a resonance that occurs at an angle oblique to the magnetic field lines. The obliqueness of the resonance angle means that both parallel $\left(n_{\|}\right)$and perpendicular $\left(n_{\perp}\right)$ components of the refrartive index vector must tend to infinit, at the resonance. The oblique resonance exists (neary) everywhere within the plasma, but occurs at a specific resonance angle determined by plasma parameters at each location. The resonance angle is larger at higher dimensionless "densities." and tends to a right angle as the lower hybrid resonance condition is approached.

Whether or not the oblique resonance can actually occur depends on the C.e. relation invoked in the analysis. In the widely employed classical accessibility analysis, the (i.C. relation is the invariance of $n_{\|}$postulated as a boundary conclition. 'The oblique resonance is thus precluded from the outset, and the lower hybrid resonance is the only resonance relevant in the analysis. Perhaps for this reason. the notion of the oblique resonance has remained obscure. But the lower hybrid resonance is a high density limit of the oblique resonance that occurs exactly at a right angle to the magnetic field lines. In the 'generalized' accessibility analysis, a less stringent constraint is placed upon $n_{\|}$. The oblique resonance nay thus occur, and alter qualitatively the nature of wave propagation. Two different C.('. relations have been studied in the context of the generalized accessibility. $A$ 'traditional' (i.C. relation is a global constraint used extensively in ray tracing calculations, and assumes the invariance 
of the toroidal mode number. A 'new' G.C' relation proposed in this article postulates the invariance of the component of the refractive index vector in a direction perpendicular to the local density gradient. Each of these assumptions leads to the invariance of a different linear combination of $n_{\|}$and $n_{\perp}$.

Solving the dispersion relation and a G.C. relation simultaneously, but without specifying the azimuthal angle (about the local magnetic field line) of wave propagation, leads to the notion of 'admissible' solutions. Many qualitative but useful understanding of wave propagation can be obtained from an examination of adnissible solutions: for example. limits of shifting of $n_{\|}$, either upward or downward, can be assessed from them. Either (i.C. relation allows $n_{\|}$ shifting. The physical basis for $n_{\|}$shifting is the pitch angle of magnetic field lines for the 'traditional' G.C. relation, and is the density gradient along the field lines for the 'new' G.C. relation. When the oblique re sonance is realizable, $n_{\|}$upshifting can br unlimited, and toroidal reflection of waves may also occur. When the oblique resonance is not realizable, upshifting is bounded, and toroidal reflection cannot occur.

It is commonly believed that lower hybrid waves can damp in spite of spectral gaps, because $n_{\|}$upshifting bridges them. Large spectral gaps cannot. however, be bridged by $n_{\|}$upshifting, if it is to be bounded. Wave damping through $n_{\|}$upshifting should not then occur in the presence of a large enough spectral gap. It was shown through an analysis using model plasmas that this theoretical expectation leads to contradiction to experiment al evidence. if the 'traditional' (i. $($ '. relation is employed. For the TRIAM model plasma. the slowest possible waves were predicted to propagate at speeds fourteen times the electron thermal speed. and a large unbridgeable spectral gap existed. In the case of PBX-M tokamak. there were conditions under which "\| upshifting was predicted to be too severely limited to bridge the spectral gap. but experimental evidence reported elsewhere ${ }^{17}$ showed that a current was driven by L.H('1). Thus, "y upshifting through the pitch angle of field lines cannot be the only mechanism that lear!s to lower hybrid wave damping, and may possibly be a mechanism inapplicable to these experiments.

Parallel density gradients may be present in the equilibrium state under special circumstances, or may be produced by commonly observed density fluctuatiuns. The proposed $n_{\|}$ upshifting mechanism based upon the parallel density gradient is a candidate to explain experimentally observed lower hybrid wave damping in the face of speetral gaps.

Past ion heating and current drive experiments. and more recent profile modification experiments have left some fundamental issues of lower hybrid wave physics unresolved. They include the lack of consistent observation of ion heating. and the existence of a density limit in the current drive efficacy. In connection with possible mechanisms to fill the spectral gap. the existence of seed electrons' is often postulated. but the origin of these electenns has remained unspecified. The proposed $n_{\|}$upshifting mechanism may offer plansible quninative explanations to these issues. The following discussion in the remainder of this section is in part based upon findings of the present article and in part on conjecture.

Lower hybrid waves remain perfectly coherent in the toroidal direction in an idealized tokamak with a perfect axisymmetry, and the toroidal mode number may be taken as strictly invariant. Density fluctuations may reduce the coherence in real tokamaks, and the global 
constraint relation may become less strictly applicable. In the presence of fluctuations of larger amplitudes, a local constraint relation may become more appropriate. Density fluctuations have been measured in tokamaks ${ }^{30-32}$ with relative amplitude ranging from a fraction of one percent to several tens of percent, depending upon the radial location within the plasma and the size of the tokamak. The parallel gradient has never been measured, but is theoretically believed to be quite small. Nevertheless, large tilting of the density gradient can occur wherever the perpendicular gradient is significantly reduced or vanishes. We will discuss below how the proposed upshifting mechanism, if it indeed occurs, may be able to explain qualitatively some of the unresolved issues.

Consider a toroidal geometry with or without poloidal magnetic fields. The density is high enough to satisfy the lower hybrid resonance condition at an interior point, and density fluctuations also exist. Fluctuations of ever smaller amplitudes are then suffice to satisfy the unbounded $n_{\|}$upchifting condition (i.e., the tilt angie equals $\pi / 2-\zeta_{\text {res }}$ ) as the lower hybrid resonance layer is approached. Unbounded upshifting leads to strong electron Landau damping. Waves may then be damper out by electron Landau damping before they come sufficiently close to the lower hybrid layer to be damped by perpendicular ion Landau damping or to be absorbed through mode conversion. The proposed $n_{\|}$upshifting mechanism is thus not incrasistent with the lack of observation of reproducible ion heating and with the lack of observation ${ }^{3}$ of mode conversion products.

The density limit of LHCD has been observed ${ }^{\top}$ in many tokamaks. Several theses have been advanced to explain the limit, for example, in terms of mode conversion to warm plasma waves by Sverdrup and Bellan, ${ }^{33}$ and in terms of wave absorption hy a minority species by Alladio. et al. ${ }^{34}$ We propose here that toroidal reflection of waves may play a role in reducing the current drive efficacy at high densities: fluctuations of smaller amplitudes will suffice to satisfy the condition for toroidal wave reflection.

It was reported by McWilliams, et al., ${ }^{35}$ that strong lower hybrid wave damping was observed in the IRVINE TORUS device in the presence of a large spectral gap and strong density fluctuations $\left(\delta n_{\epsilon} / n_{,} \sim 0.1\right)$. The device had no rotational transform, and the spectral gap could not have been bridged by the 'traditional' $n_{\|}$upshifting mochanism. The experiment was conducted close to the lower hybrid resonance, and the density gradient tilt angle required to produce unbounded $n_{\|}$upshifting was therefore small. A fluctuating parallel density gradient that is only about two percent of the equilibrium radial density gradient would be enough to create such a tilt angle, even no reductions in the perpendicular gradient occur. The propsed mechanism is thus not inconsistent with the experimental observations. (Using reported numbers, $R_{0}=0.556 \mathrm{~m}, a_{p}=0.1 \mathrm{~m}, B_{\phi}=0.1 \mathrm{~T}, \omega=2 \pi \times 70 \mathrm{MHz}, n_{\phi}^{g r}=14.3$, and under the assumption of a helium plasma with a parabolic density profile with $n_{f}(0)=3 \cdot 10^{18} / \mathrm{m}^{3}$, we obtain $\omega_{L H} / \omega \sim 0.65$ and $\zeta_{\text {res }} \sim 88.9 \mathrm{deg}$ at the plasma center. When the density gradient tilt angle, $a=2$ deg. is assumed. Figs. 24 through 26 describe conditions in IRVINE TORUS at $1 \mathrm{~mm}, 1.5 \mathrm{~mm}$ and $4 \mathrm{~mm}$ from the plasma edge, respectively. Interior points are qualitatively similar to the last case.)

In discussion of spectral gap issues, the notion of 'seed electrons' is common. If there

\footnotetext{
${ }^{7}$ See an article by Sverdrup and Bellan ${ }^{33}$ for an extensive list of earlier publications on this subject.
} 
is a 'tail' electron population, no matter how small, that fills the spectral gap between the thermal electron population and the wave spectrum, waves will deplete the population by carrying away the tail electrons to higher energies. The tail population is then replenished from the thermal population. The process thus effectively keeps 'drawing out a tail' of the thermal population. An important 'gap' in this explanation is the fact that the source of the original tail electron population is unspecified. The proposed $n_{\|}$upshifting mechanism is compatible with generation of such seed electrons. Fluctuations of a large 'amplitude' (in the sense of creating a strong density gradient tilt angle), which may occur only infrequently and at discrete spatial locations, can cause unbounded $n_{\|}$upshifting, temporarily and locally, in otherwise bounded upshifting circumstances. Strong upshifting causes waves to interact with the thermal population, and create a tail population. Such tail electrons may survive for a finite time period after the large-amplitude fluctuations disappear, and may also be transported away from their birth places. Those waves that experience only bounded $n_{\|}$upshifting can now interact with the tail electrons.

There may be other circumstances in which a strong parallel density gradient is generated in the equilibrium state. For example, a $\operatorname{Marfe}^{36}$ is a poloidally localized region of high densities, and there are parallel density gradients along field lines that enter the region. Interaction of an LHCD antenna with the plasma may also generate a localized region of altered densities in the immediate vicinity of the structure: a ponderomotive force of the antenna near-field may generate a region of lower densities, ${ }^{3 \tau}$ leading to a finite parallel gradient and at the same time to a reduced perpendicular gradient, or arcing may generate a region of higher densities.

The proposed $n_{\|}$upshifting mechanism must be corroborated by experimental evidence. Interpretation of experimental results will require measurements of fluctuations and statistical analysis tools. Scattering of lower hybrid waves by density fluctuations has been considered analytically by Andrews and Perkins, ${ }^{38}$ and has recently been iiclisded in ray tracing calculations by Vahala, et al., ${ }^{39}$ and by Ignat, et al. ${ }^{40}$ In such an analysis, the choice of 'boundary conditions' is crucial: wave scattering while preserving the toroidal mode number will lead to a test of the 'traditional' upshifting mechanism. and wave scattering while preserving the component of the refractive index vector in a direction petpendicular to the density gradient will lead to a test of the 'new' upshifting mechanism.

Finally, efforts to resolve spectral gap issues must include effects ot her than $n_{\|}$upshifting. The spectral gap is room in velocity space between the wave spectrum and (a few times) the parallel electron thermal speed. Room can be filled with waves by extending downward the imposed wave spectrum, as is thought to occur in the $n_{\|}$upshifting hypothesis, or it can be filled by electrons brought in from elsewhere in velocity space by some mechanism. The reverserunning portion of a grill-imposed spectrum has been proposed by ' 'akahashi, et al. ${ }^{17,26}$ and by Colborn, et al., ${ }^{41}$ as a possible mechanism to fill with fast elcctrons the spectral gap that remains unbridged after the maximum possible $n_{\|}$upshifting. Any role played by suprathermal electrons that may naturally be present in tokamak plasmas should also be investigated. ${ }^{42}$ It is also noted that the applicability of the ray tracing theory itself to lower hybrid waves has been examined by Pereverzev. ${ }^{43}$ 


\section{ACKNOWLEDGMENT}

The author would like to express his gratitude to Drs. M. Ono and D. Ignat for many valuable discussions. He would also like to thank Drs. S. Bernabei, T.K. Chu, R. Kaita, S. Parker, F. Perkins, T. Stix and S. von Goeler for helpful discussions. This work was supported by U.S. Department of Energy under Contract No. DE-AC02-76-CHO-3073. 


\section{References}

${ }^{1}$ N. Fisch, Phys. Rev. Lett., 41, 873, (1978).

${ }^{2}$ P. Bonoli, IEEE Transactions on Plasma Science, PS-12(1984)95.

${ }^{3}$ M. Porkolab, IEEE Transactions on Plasma Science, PS-12(1984)107.

${ }^{4}$ N. Fisch, Rev. Mod. Physics, 59(1987)175.

${ }^{5}$ C. Gormezano, et al., Proc. of the 18th EPS Conf. on Controlled Fusion and Plasma Physics (Berlin, 1991), Vol.15C, Part-III, Paper E37, pIII-393, European Physical Society.

${ }^{6}$ F. Soeldner, et al., in Plasma Physics and Controlled Nuclear Fusion Research 1990 (IAEA, Vienna, 1991), Vol. I, p613.

${ }^{7}$ S. Bernabei, et al., Phys. Fluids, B 5(1993)2562.

${ }^{8}$ R. Bell, et al., 10th Topical Conf. on Radio Frequency Power in Plasmas, Invited Paper C11, Boston, April, 1993.

${ }^{9} \mathrm{~K}$. Ushigusa, et al., Proc. of the 20th EPS Conf. on Controlled Fusion and Plasma Physics (Lisbon, 1993), Vol.17C, Part-III, Paper 5-17, p913, European Physical Society.

${ }^{10}$ J. Schuss, et al., Nucl. Fusion, 21, 427, (1981).

${ }^{11}$ J.-M. Wersinger, E. Ott, and J. Finn., Phys. Fluids, 21(1978)2263.

${ }^{12}$ E. Ott, J.-M. Wersinger, and P. Bonoli., Phys. Fluids, 22(1979)192.

${ }^{13}$ D.W. Ignat, Phys. Fluids, 24(1981)1110.

${ }^{14} \mathrm{P}$. Bonoli and E. Ott, Phys. Fluids, 25(1982)359.

${ }^{15}$ P. Bonoli and R. Englade, Phys. Fluids, 29(1986)2937.

${ }^{16}$ K. Kupfer and D. Moreau, Nucl. Fusion, 32(1992)1845.

${ }^{17} \mathrm{H}$. Takahashi, et al., Proc. of the 20th EPS Conf. on Controlled Fusion and Plasma Physics (Lisbon, 1993), Vol.17C, Part-III, Paper 5-14, p901, European Physical Society.

${ }^{18}$ R.J. Briggs and R.R. Parker, Phys. Rev. Lett., 29, $852-855$ (1972).

${ }^{19}$ F. Paoletti, et al., submitted to Nucl. Fusion.

${ }^{20} \mathrm{~T}$. Yamagajo, et al.,Proc. of the 20th EPS Conf. on Controlled Fusion and Plasma Physics (Lisbon, 1993), Vol.17C, Part-III, Paper 5-15, p905, European Physical Society.

${ }^{21}$ T. H. Stix, The Theory of Plasma Waves, McGraw-Hill, New York, 1962.

${ }^{22}$ T. H. Stix, Waves in Plasmas, American Institute of Physics, New York, 1992. 
${ }^{23}$ T. H. Stix, Phys. Rev. Lett., 15(1965)878.

${ }^{24}$ M. Brambilla, Nucl. Fusion, 16(1976)47.

${ }^{25}$ W. P. Allis, S. J. Buchsbaum and A. Bers, Waves in Anisotropic Plasmas, The M.I.T. Press, Cambridge, Massachusetts, 1963.

${ }^{26}$ H. Takahashi, R. Harvey, S. Bernabei, A. England, D. Ignat, and S. von Goeler, Paper 8T5, Bull. Am. Phys. Soc., Series II, Vol. 38, p2092, Nov., 1993.

${ }^{27}$ S. Bernabei, et al., Nucl. Fusion, 26, 111-114, (1986).

${ }^{28}$ P.M. Bellan and M. Porkolab, Phys. Fluids, 19, 995-1005 (1976).

${ }^{29}$ S. Parker, W. Lee, and R. Santoro, Phys. Rev. Lett., 71, 2042-2045 (1993).

${ }^{30}$ R. Slusher and C. Surko, Phys. Rev. Lett., 40, 400-403 (1978).

${ }^{31}$ T. Crowley and E. Mazzucato, Nucl. Fusion, 25, 507-524 (1985).

${ }^{32}$ R. Fonck, et al., Phys. Rev. Lett., 70, 3736-3739 (1993).

${ }^{33}$ L.H. Sverdrup and P.M. Bellan, Phys. Rev. Lett., 59, 1197-1200 (1987).

${ }^{34}$ F. Alladio, et al., Nucl. Fusion, 24, 725-731 (1984).

${ }^{35}$ R. McWilliams, US-Japan Workshop on Non-Inductive (urrent Drive and Profile Control, Princeton, NJ, Dec., 1992.

${ }^{36}$ B. Lipschultz, et al., Nucl. Fusion, 24977 (1984).

${ }^{37}$ T.K. Chu, private communication.

${ }^{38}$ P.L. Andrews and F.W. Perkins, Phys. Fluids, 26(1983)2537 and 26(1983)2546.

${ }^{39}$ G. Vahala, L. Vahala, and P.T. Bonoli, Phys. Fluids, B4(1992)4033.

${ }^{40}$ D.W. Ignat, E.J. Valeo and S.C. Jardin, submitted to Nucl. Fusion; also, Princeton Plasma Physics Laboratory Report, PPPL-2929, Oct., 1993.

${ }^{41}$ J.A. Colborn, et al., Submitted to Phys. Fluids.

${ }^{42} \mathrm{~S}$. von Goeler, private communication.

${ }^{43}$ G.V. Pereverzev, Proc. of the 20th EPS Conf. on Controlled Fusion and Plasma Physics (Lisbon, 1993), Vol.17C, Part-III, Paper 5-10, p885, European Physical Society. 
Dr. F. Paoloni, Univ. of Wollongong, AUSTRALIA

Prof. M.H. Brennan, Univ. of Sydney, AUSTRALIA

Plasma Research Lab., Australian Nat. Univ., AUSTRALIA

Prof. I.R. Jones, Flinders Univ, AUSTRALIA

Prof. F. Cap, Inst. for Theoretical Physics, AUSTRIA

Prof. M. Heindler, Institut für Theoretische Physik, AUSTRIA

Prof. M. Goossens, Astronomisch Instituut, BELGIUM

Ecole Royale Militaire, Lab. de Phy. Plasmas, BELGIUM

Commission-European, DG. XII.Fusion Prog., BELGIUM

Prof. R. Bouciqué, Rijksuniversiteit Gent, BELGIUM

Dr. P.H. Sakanaka, Instituto Fisica, BRAZIL

Prof. Dr. I.C. Nascimento, Instituto Fisica, Sao Paulo, BRAZIL

Instituto Nacional De Pesquisas Espaciais-INPE, BRAZIL

Documents Office, Atomic Energy of Canada Ltd., CANADA

Ms. M. Morin, CCFMTokamak de Varennes, CANADA

Dr. M.P. Bachynski, MPB Technologies, Inc., CANADA

Dr. H.M. Skarsi urd, Univ. of Saskatchewan, CANADA

Prof. J. Teichmann, Univ. of Montreal, CANADA

Prof. S.R. Sreenivasan, Univ. of Calgary, CANADA

Prof. T.W. Johnston, INRS-Energie, CANADA

Dr. R. Bolton, Centre canadien de fusion magnétique, CANADA

Dr. C.R. James, Univ. of Alberta, CANADA

Dr. P. Lukác, Komenského Universzita, CZECHO-SLOVAKIA

The Librarian, Culham Laboratory, ENGLAND

Library, R61, Ruthertord Appleton Laboratory, ENGLAND

Mrs. S.A. Hutchinson, JET Library, ENGLAND

Dr. S.C. Shama, Univ. of South Pacific, FIJI ISLANDS

P. Mähönen, Univ. of Helsinki, FINLAND

Prof. M.N. Bussac, Ecole Polytechnique., FRANCE

C. Mouttet. Lab. de Physique des Milieux lonisés, FRANCE

J. Radel, CEN/CADARACHE - Bat 506, FPANCE

Prof. E. Economou, Univ. ol Crete, GREECE

Ms. C. Rinni, Univ, of loannina, GREECE

Preprint Library, Hungarian Academy of Sci., HUNGARY

Dr. B. DasGupta, Saha Inst. of Nuclear Physics, INDIA

Dr. P. Kaw, Inst. for Plasma Research, INDIA

Dr. P: Rosenau, Israel Inst. of Technology, ISRAEL

Librarian, Intemational Center for Theo Physics, ITALY

Miss C. De Palo, Associazione EURATOM-ENEA , ITALY

Dr. G. Grosso, Istituto di Fisica del Plasma, ITALY

Prof. G. Rostangni, Istituto Gas Ionizzati Del Cnr, ITALY
Dr. H. Yamato, Toshiba Res \& Devel Center, JAPAN

Prof. I. Kawakami, Hiroshima Univ., JAPAN

Prof. K. Nishikawa, Hiroshima Univ., JAPAN

Librarian, Naka Fusion Research Establishment, JAERI, JAPAN

Director, Japan Atomic Energy Research Inst., JAPAN

Prot. S. Itoh, Kyushu Univ., JAPAN

Research Info. Ctr., National Instit. for Fusion Science, JAPAN

Prol. S. Tanaka, Kyoto Univ., JAPAN

Library, Kyoto Univ., JAPAN

Prof. N. Inoue, Univ. of Tokyo, JAPAN

Secretary, Plasma Section, Electrotechnical Lab., JAPAN

S. Mori, Technical Advisor, JAERI, JAPAN

Dr. O. Mitarai, Kumamoto Inst. of Technology, JAPAN

Dr. G.S. Lee, Korea Basic Sci. Ctr., KOREA

J. Hyeon-Sook, Korea Alomic Energy Research Inst., KOREA

D.I. Choi, The Korea Adv. Inst. of Sci. \& Tech., KOREA

Prof. B.S. Liley, Univ. of Waikato, NEW ZEALAND

Inst of Physics, Chinese Acad Sci PEOPLE'S REP. OF CHINA

Library, Inst. of Plasma Physics, PEOPLE'S REP. OF CHINA

Tsinghua Univ. Library, PEOPLE'S REPUBLIC OF CHINA

Z. L. S.W. Inst Physics, PEOPLE'S REPUBLIC OF CHINA

Prof. J.A.C. Cabral, Instituto Superior Tecnico. PORTUGAL

Prof. M.A. Hellberg, Univ. of Natal, S. AFRICA

Prof. D.E. Kim, Pohang inst. of Sci. \& Tech., SO. KOREA

Prof. C.I.E.M.A.T, Fusion Division Library, SPAIN

Dr. L. Stenflo, Univ. of UMEA, SWEDEN

Library, Royal Inst. of Technology, SWEDEN

Prof. H. Wilhelmson, Chalmers Univ. of Tech., SWEDEN

Centre Phys. Des Plasmas, Ecole Polytech, SWITZERLAND

Bibliotheek, Inst. Voor Plasma-Fysica, THE NETHERLANDS

Asst. Prof. Dr. S. Cakir, Middle East Tech. Univ., TURKEY

Dr. V.A. Glukhikh,Sci. Res. Inst. Electrophys.I Apparatus, USSR

Dr. D.D. Ryutov, Siberian Branch of Academy of Sci., USSR

Dr. G.A. Eliseev, I.V. Kurchatov Inst., USSR

Librarian, The Ukr.SSR Academy of Sciences, USSR

Dr. L.M. Kovrizhnykh, Inst. of General Physics, USSR

Kemforschungsanlage GmbH, Zentralbibliothek, W. GERMANY

Bibliothek, Inst. Für Plasmaforschung. W. GERMANY

Prof. K. Schindler, Ruhr-Universitát Bochum, W. GERMANY

Dr. F. Wagner, (ASDEX), Max-Planck-Institut, W. GERMANY

Librarian, Max-Planck-Institut, W. GERMANY 


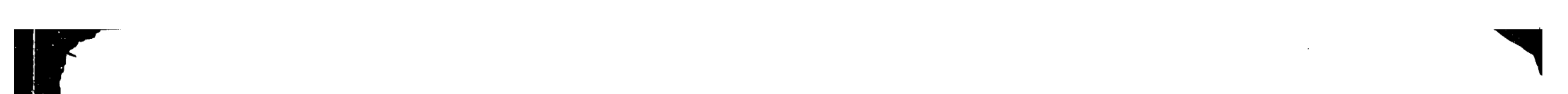


